\title{
Atomic Chains at Surfaces
}

\author{
J. E. Ortega ${ }^{1,2}$ and F. J. Himpsel ${ }^{3}$ \\ 1 Departamento de Física Aplicada I, Universidad del País Vasco, Plaza de Oñate \\ 2, E-20018 San Sebastian, Spain enrique.ortega@ehu.es \\ 2 DIPC and Centro Mixto CSIC/UPV, Paseo Manuel Lardizabal 4, E-20018 San \\ Sebastian, Spain \\ 3 Department of Physics, University of Wisconsin Madison, 1150 University Ave., \\ Madison, Wisconsin 53706, USA fhimpsel@facstaff.wisc.edu
}

\section{Introduction to One-Dimensional Systems}

\subsection{Physics in one dimension}

One-dimensional (1D) physics is particularly elegant and simple. Many problems can be solved analytically in one dimension, but not in higher dimensions. Some problems can only be solved in one dimension. 1D is the lowest dimension, where electrons are able to propagate. In that sense, 1D is the lowest non-trivial dimension. For these reasons, quite a few books have been written about physics in one dimension $[1,2]$.

It has been predicted for some time that electrons exhibit fundamentally different properties when confined to move along a single dimension. Singleparticle excitations are replaced by collective excitations since the electrons cannot avoid each other when moving along a single line. Their wave functions overlap completely and become highly correlated. This strong interaction has startling consequences on the physics of one-dimensional systems leading to a variety of unusual phases at low temperatures $[3,4,5,6,7]$. In a onedimensional metal even the identity of electrons is lost, and becomes replaced by separate spin and charge excitations, the spinons and holons $[3,4,5]$. The chapter by Claessen et al. discusses these phenomena in detail. While the predictions give glimpses of exotic physics, finding direct evidence for spin-charge separation has proven elusive. It has been very difficult to systematically tailor natural crystals with 1D character, such that the electronic properties can be optimized for reaching the appropriate part of the 1D phase diagram and avoiding other low-dimensional instabilities, such as a Peierls transition to an insulator, charge density waves, spin density waves, singlet and triplet superconductivitiy, etc. [6, 7]. 


\subsection{Creating 1D structures}

While theorists have it easier in 1D, experimentalists have to work harder. In theory, the ideal 1D system is a string of atoms freely suspended in space. Experimentally, that is only possible for strings of a few atoms $[8,9]$, and these atoms vibrate enormously. Nevertheless, there have been encouraging developments in surface physics, where stepped surfaces or otherwise anisotropic surfaces have been used as templates for creating 1D structures.

Particularly interesting are metallic chain structures on semiconductor surfaces [10]. The atoms are locked rigidly to the substrate by covalent bonds, but the electrons near the Fermi level completely de-couple from the substrate. They cannot hybridize with any of the three-dimensional bulk states, as long as they lie in the band gap of the substrate. Only the electrons in back-bond states are able to hybridize, but these lie well below the Fermi level (typically $5-10 \mathrm{eV}$ ). The dimensionality can be controlled by adjusting the step spacing. 1D/2D coupling ratios ranging from 10:1 to well above 70:1 have been achieved $[11,12]$. The surface band structure exhibits unexpected features, such as a fractional electron count per chain atom, two half-filled bands instead of one completely filled band, and nanoscale phase separation into metallic and insulating chain sections [13]. Spin chains can be created by using transition metal and rare earth atoms [14, 15], and the orbital and spin moment changes dramatically in these structures.

While semiconductor surfaces provide a variety of exotic phenomena, they are more difficult to understand because of the complicated rearrangement of their broken bonds, which may involve more than hundred atoms, such as in the famous $\operatorname{Si}(111) 7 \times 7$ surface, the most stable surface of silicon. Therefore, it is advisable to begin with metal surfaces, where the bonding is isotropic and extensive rearrangements are rare. The electronic states are delocalized and free-electron-like, in contrast to the strongly localized broken bond orbitals of semiconductors. Stepped metal surfaces will serve us as textbook examples for explaining the type of low-dimensional states that can be induced by steps.

\subsection{Mapping 1D electrons:}

Angle-resolved photoemission has the distinction of being capable to determine the complete set of quantum numbers for electrons in a solid and at a surface. These are energy, momentum, spin, and the point group symmetry. In a $2 \mathrm{D}$ system, both in-plane momentum components are conserved in the photoemission process up to a reciprocal lattice vector. Therefore, measuring the energy and the in-plane momentum of the emitted photoelectron provides directly the energy and momentum of the electron inside the solid using energy and momentum conservation. The resulting data set consists of the photoemission intensity as a function of three variables, the "intensity curve" $I\left(E, k_{x}, k_{y}\right)$. Typically, two-dimensional slices of this cube are plotted on a gray scale or in color code. (A popular scheme is that of a topographic map, 
where the deep blue sea represents lowest intensity and white mountains the highest.) The $I\left(E, k_{x}\right)$ and $I\left(E, k_{y}\right)$ slices represent the familiar $E(k)$ band dispersion, while the $I\left(k_{x}, k_{y}\right)$ slices are constant energy "surfaces". The most important of these is the Fermi surface, where the electronic states reside that are responsible for most of the interesting electronic phenomena, such as charge density waves, magnetism, superconductivity, and transport in general. An example is shown in Fig. 1, where the two types of slices are combined for a nearly one-dimensional chain structure on silicon.

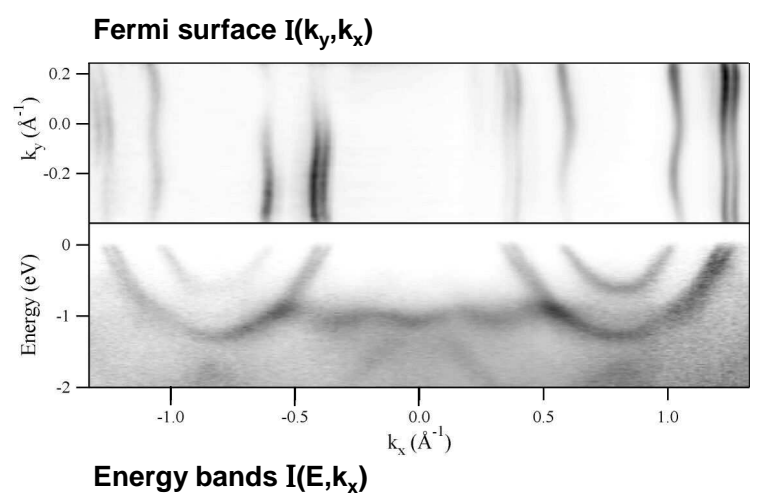

Fig. 1. Two ways of displaying an angle-resolved photoemission data set, demonstrated for the one-dimensional chain structure of Si(553)-Au (from [11]).

Bottom: Photoemission intensity versus energy $E$ and wave vector $k_{x}$ (with $k_{x}$ in the chain direction), representing a band dispersion. The metallic bands crossing the Fermi level $E_{F}$ are free-electron-like, i.e., nearly parabolic.

Top: Photoemission intensity at $E_{F}$ versus $k_{y}$ and $k_{x}$, representing the Fermi surface. High photoemission intensity is shown dark.

Comparing Fermi surfaces of two- and one-dimensional structures in Fig. 2 illustrates the dramatic effect of dimensionality on the topology of the Fermi surface. In 3D, the Fermi surface of a free electron is a sphere with radius $k_{F}$, in $2 \mathrm{D}$ a circle with radius $k_{F}$, and in $1 \mathrm{D}$ it degenerates into two points at $\pm k_{F}$. By measuring a $1 \mathrm{D}$ system embedded into a $2 \mathrm{D}$ surface, the two $k_{F}$ points become spread out into lines perpendicular to the $1 \mathrm{D}$ atom chain. This embedding process is analogous to the mapping of a $2 \mathrm{D}$ surface diffraction pattern in $3 \mathrm{D}$ space, which leads to a spreading of the 2D Bragg spots into "rods' perpendicular to the surface. In the example in Fig. 2, the lines are not completely straight, which indicates a residual 2D coupling between 1D chains.

While high energy resolution is always a benefit for resolving sharp states at the Fermi level, even more important for 2D and $1 \mathrm{D}$ systems at surfaces is $k$-resolution. The unit cells on semiconductors are rather large in real space, such as the $7 \times 7$ cell. De-coupling the wires in a surface arrays requires a 


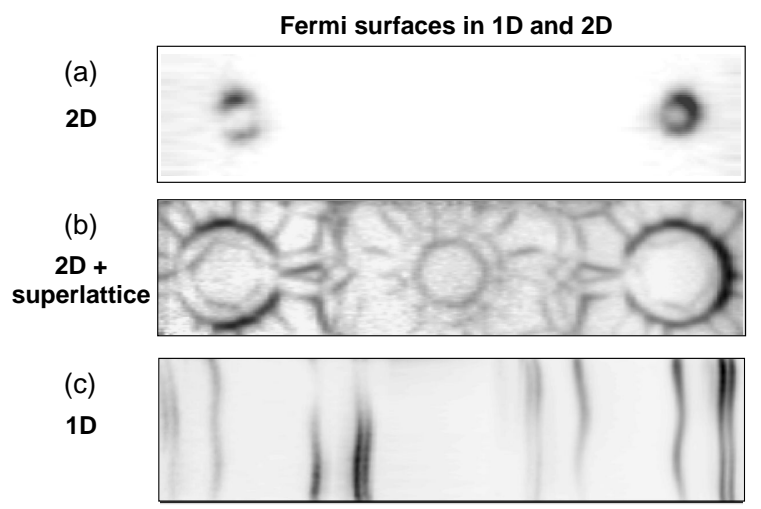

Fig. 2. Comparison between Fermi surfaces in 2D an 1D. The topology of the Fermi surface changes completely from circles to lines. Having a superlattice creates replicas of the the Fermi circles that are shifted reciprocal superlattice vectors. a) $\mathrm{Si}(111) \sqrt{3} \times \sqrt{3}-\mathrm{Ag}, \mathrm{b}) \mathrm{Si}(111) \sqrt{21} \times \sqrt{21}-(\mathrm{Ag}+\mathrm{Au}), \mathrm{c}) \mathrm{Si}(553)-\mathrm{Au}$. High photoemission intensity is shown dark. From [16].

large wire spacing, which leads to a small Brillouin zone perpendicular to the wires.

\section{One-Dimensional Quantum Wells at Metal Surfaces}

Recent developments in high energy and angular resolution in photoemission have an enormous impact on the study of electronic states of low-dimensional nanostructures on metal surfaces, which are readily accessible by this technique. Noble metal surfaces, in particular, offer a relatively simple electronic structure, which has been investigated in great detail by photoemission and scanning tunneling spectroscopy. As a consequence, there is a wealth of data and a detailed knowledge of the electronic properties for a variety of metallic surfaces. Knowing the electronic states of a flat surface is crucial for disentangling the complexity that arises in photoemission experiments from lateral nanostructures grown on top the surface. Low-dimensional structures on well-known metal surfaces are very valuable as model systems to investigate electronic states with angle-resolved photoemission. Additionally, one can envision potential applications for magnetic quantum stripes and atom chains [14], or for nanostructured metallic templates, which can be used for selective chemical adsorption in molecule/metal systems $[17,18]$, such as those searched for in catalysis, solar cell, or light emitting diode device technologies.

\subsection{The vicinal noble metal surface as a model 1D system}

One of the simplest nanostructured metallic systems is a vicinal surface with a regular array of monatomic steps, which give the surface a one-dimensional 
(1D) character. Vicinal surfaces are miscut by a few degrees from a high symmetry direction and can be readily prepared in-situ, with the standard procedure of ion sputtering and mild annealing. The quality of the resulting step superlattice depends on a variety of factors, such as surface energy versus step and kink energy. Homogeneous arrays of steps with lattice constant $d$ are frequently obtained over micron-size patches of the surface. Nonetheless, such well-defined areas are always characterized by a finite terrace size distribution $\sigma=\Delta d / d$, which in turn varies upon the strength of the interaction between steps [19]. The size distribution $\sigma$ in lateral nanostructures and arrays selfassembled on solid surfaces is a key parameter that is necessary to control, since it introduces an intrinsic spectral broadening in averaging techniques, such as angle resolved photoemission.

(a)

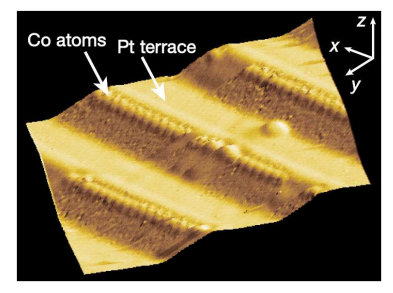

(b)

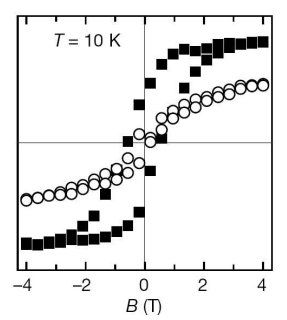

Fig. 3. (a) STM topography showing $1 \mathrm{D}$ atomic chains of Co grown by step decoration on $\mathrm{Pt}(997)$. (b) The magnetization of such chains is measured along the easy (filled symbols) and hard (open symbols) directions. The magnetic response at $\mathrm{T}=$ $10 \mathrm{~K}$ reveals non-zero remanent magnetization and hence long-range ferromagnetic order (from [14]).

Vicinal surfaces are particularly useful as templates for the self-assembly of 1D metallic nanostructures. The general strategy consists on depositing submonolayer amounts of distinct materials from the gas phase under the right conditions (temperature and flux) to promote rapid diffusion on the surface and sticking to the steps. Ideally, one-dimensional arrays of linear structures of varying thickness are produced in the row-by-row, or "step-flow" growth regime. Thickness control is not an issue, since fine tuning is readily achievable by smoothly varying the coverage across a macroscopic sample. The ultimate limit is the atomic chain, like the one shown in Fig. 3 (a). These are 1D Co atomic chains deposited on the stepped $\operatorname{Pt}(997)$ surface [14]. The reduced atomic coordination of the monatomic chains causes a remarkable increase of the magnitude of both the orbital and the spin magnetic moments compared to bulk Co. The orbital magnetic moment increases from $0.15 \pm 0.01 \mu_{B}$ /atom in the bulk-like Co film $0.68 \pm 0.05 \mu_{B}$ /atom in the monatomic chain. Moreover, as demonstrated by the hystheresis cycle in Fig. 3 (b), such Co chains sustain long-range ferromagnetic order. That is possible thanks to the strong 
magnetic anisotropy energy barriers, which effectively block the relaxation of the magnetization at sufficiently low temperature.

(a)
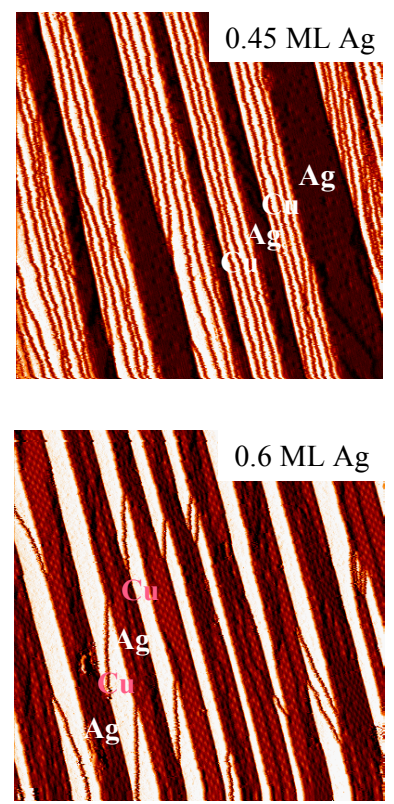

(b)
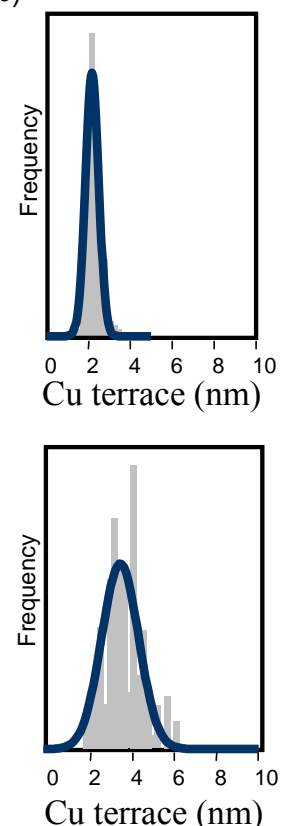

(c)
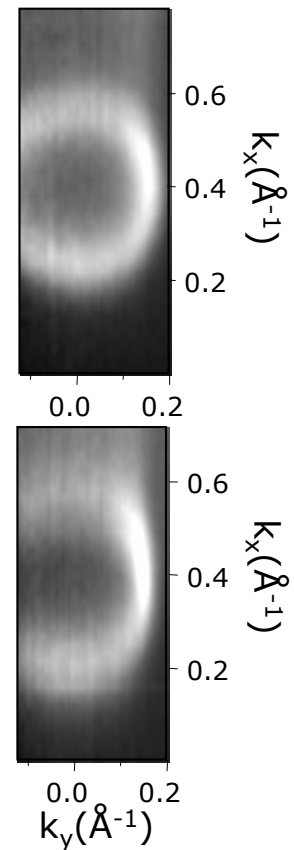

Fig. 4. One-dimensional periodic faceting in a vicinal $\mathrm{Cu}(111)$ surface induced by $\mathrm{Ag}$ adsorption. (a) STM image showing Ag covered facets that alternate with clean stepped $\mathrm{Cu}$ bands at different $\mathrm{Ag}$ thickness. The size distribution of (111)-oriented $\mathrm{Cu}$ terraces is analyzed in (b). The standard deviation $\sigma$ is found to be analogous to that of the bare $\mathrm{Cu}$ substrate. The $1 \mathrm{D}$ character of surface states in narrow $\mathrm{Cu}$ nanostripes is shown in their Fermi surface in (c), which display strong asymmetry with large $k_{x}$ broadening perpendicular to the stripes (adapted from [23, 25]).

The ideal step-flow regime is rather exceptional. Frequently molecules and adatoms form zero-dimensional aggregates attached to steps, and in many other cases the adsorption induces chemical as well as structural changes in the vicinal substrate, such as faceting. In the latter the surface plane contains two separated 1D phases that are subject to mutual elastic repulsion, leading to a periodic 1D, hill-and-valley pattern. Such periodically faceted structures are also attractive as 1D metallic systems. They are stiffer than bare stepped surfaces and have longer wavelengths that can even reach the mesoscopic scale. Additionally, they display an enhanced chemical contrast between phases, which is particularly useful to achieve 1D functional stripes (molecules, ferromagnets) by selective adsorption $[17,18]$. In Fig. 4 (a) we show a character- 
istic example of adsorption-induced faceting, namely the $\mathrm{Ag} / \mathrm{Cu}$ system. The faceted structure is formed by depositing submonolayer amounts of $\mathrm{Ag}$ on the vicinal $\mathrm{Cu}(335)$ substrate at $300 \mathrm{~K}$, and then post-annealed at $450 \mathrm{~K}$ [20]. By varying the amount of $\mathrm{Ag}$, the width of the stripes and the periodicity of the system can be changed. Despite the complexity of this self-assembly process, it does not show appreciable kinetic constraints, since the size distribution in Fig. 4 (b) is analogous to that of the $\mathrm{Cu}(335)$ vicinal substrate. Such a sharp size distribution makes angle-resolved photoemission studies of surface states meaningful. It is indeed observed that distinct $\mathrm{Ag}$ and $\mathrm{Cu}$ states characterize the two phases of the system [21, 22]. The $\mathrm{Cu}$ stripe thickness determines its surface state dimensionality, which is observed to vary from $2 \mathrm{D}$ in relatively wide stepped stripes to $1 \mathrm{D}$ in narrow stripes. The 1D character in the latter is reflected in the large asymmetric broadening of the Fermi surface shown in Fig. 4 (c).

\subsection{Complex scattering at steps: From 2D to 1D surface states}

Vicinal metal surfaces and 1D striped nanostructures grown by step decoration or by periodic faceting are becoming benchmark systems to test 1D electronic states. In particular, noble metal surfaces vicinal to the (111) plane, since these possess a free-electron-like surface state easily identified in scanning tunnelling spectroscopy and well characterized in photoemission. This surface state scatters strongly at step edges, giving rise to 1D confinement and superlattice effects (for review works see $[24,25]$ and references therein). The fundamental parameter is the characteristic size $d$ perpendicular to the step array, which corresponds to the terrace width in bare stepped surfaces or to the stripe width in nanostripes decorating steps, as shown in Fig. 5 (a). There we represent the schematic side view of the resulting 1D quantum well in the direction perpendicular to the steps $x$, featured with the additional ingredients of the real system: asymmetric uphill and downhill electron potentials, dipole-like barriers at step edges and evanescent, bulk-like wave functions in the perpendicular direction $z$. All are important to understand angle-resolved photoemission data, as we shall see later. The presence of such 1D quantum well levels in metallic nanostripes has been very well documented by STS experiments, such as those reproduced in Fig. 5 (b) [26]. The STM image above shows a $d=56 \AA$ wide trench limited by monatomic step edges on a $\operatorname{Ag}(111)$ surface. These behave as hard wall potentials for surface state electrons, leading to a succession of $1 \mathrm{D}$ QW levels that are spatially probed in the STS maps below.

In a similar way as in STS, 1D metallic QW levels and wave functions can also be probed and mapped by angle resolved photoemission in noble metal vicinal surfaces, as shown in Fig. 6 [27]. Surface state bands in $\mathrm{Au}(23$ 2321 ), i.e., a $\mathrm{Au}(111)$ vicinal surface with $56 \AA$ wide terraces, break up into three QW levels below the Fermi energy. The data in Fig. 6 (a) correspond to the second derivative of the photoemission spectra displayed in an intensity 
(a)

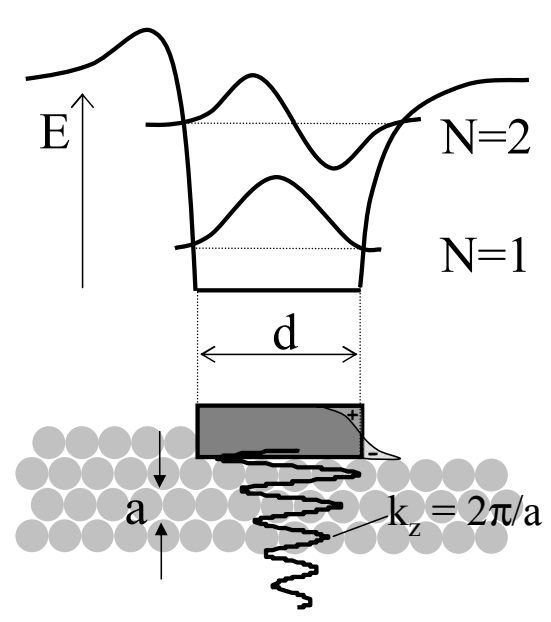

(b)

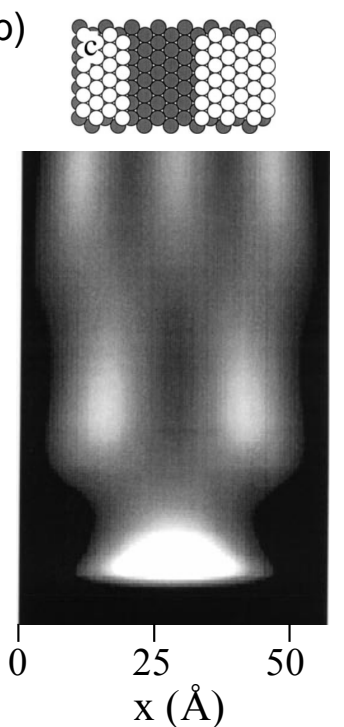

Fig. 5. (a) Schematic description of $N=1$ and $N=2$ wave functions and electron potentials for $1 \mathrm{D}$ electrons confined in a metallic nanostripe attached to a surface step. Asymmetric barriers, dipole-like step edge potentials and evanescent wave functions in the $z$ are characteristic features of the real system. Wave functions are mapped in real space by Scanning Tunneling Spectroscopy, as shown for the $\operatorname{Ag}(111)$ nanostripe in (b) (from [26]).

plot. The derivation enhances peaks while eliminating the background. The photon energy $h \nu=60 \mathrm{eV}$ is chosen to minimize the intensity from nearby umklapp features produced by photoelectron interference with the step lattice. It has been shown that the $N=1,2$, and $3 \mathrm{QW}$ levels of the figure follow the $E_{N}=E_{0}+\hbar^{2} \pi^{2} / 2 m^{*} d^{2}$ series of the infinite 1D QW of size $d=56 \AA$ [27], proving that also in the step array of the vicinal surface uphill and downhill steps may behave as hard wall potentials that confine surface electrons.

The photoemission intensity from the three QW levels of Au(23 2321$)$ is strongly modulated along the $k_{x}$ direction, as shown in Fig. 6 (b). Under certain conditions, which are met in this case, the photoemission intensity maps in Fig. 6 (b) represent the Fourier transform of the respective wave functions $\left|\Psi_{N}\left(k_{x}\right)\right|^{2}[27]$. Moreover, the confined nature of the wave function in $\mathrm{Au}(2323$ 21) terraces makes it possible to retrieve the real-space wave function $\Psi_{N}(x)$ from the experimental $\left|\Psi_{N}\left(k_{x}\right)\right|^{2}$ curve using an iterative formalism, called oversampling, borrowed from x-ray diffraction [28]. Iterative procedures overcome the well-known problem of the phase in momentum space, which is not measured in spectroscopic techniques, such as x-ray diffraction or photoemission. In iterative oversampling, the phase is obtained by repeatedly diminishing the amplitude of the wave function outside the confinement region, 

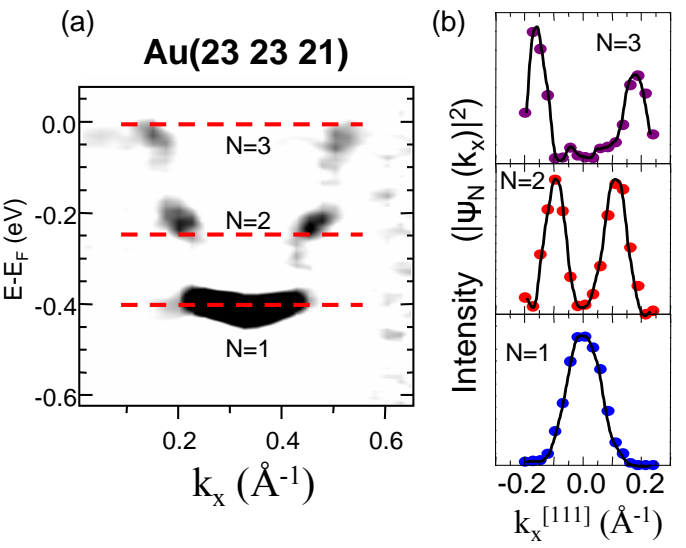

(c)

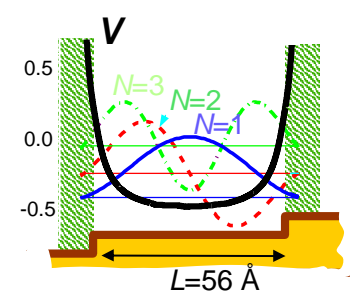

Fig. 6. Quantum wells in the $\mathrm{Au}(232321)$ observed by angular photoemission. (a) Second derivative of the photoemission spectra shown as an intensity plot. The surface band breaks up in non-dispersing levels that fit to an infinite 1D QW of size $d=56 \AA$. (b) Photoemission intensity map $\left|\Psi_{N}\left(k_{x}\right)\right|^{2}$ and (c) real space wave functions $\Psi_{N}(x)$ and effective electron potential $V(x)$ determined from an iterative phase recovery process using the data in (b). Note that $k_{x}$ is defined with respect to the average surface in (a), but with respect to the (111) terrace in (b) (from [27]).

which in turn is estimated from the self-convolution of the $\left|\Psi_{N}\left(k_{x}\right)\right|^{2}$ curve. The three QW wave functions shown in Fig. 6 (c) are obtained after applying the oversampling method to the data in Fig. 6 (b). On the other hand, the effective one-electron potential (thick line) is obtained by simply dividing the Schrödinger equation by the wave function.

In the past few years, a large number of studies have been devoted to the study of the electronic structure in vicinal noble metal surfaces using high-resolution angle-resolved photoemission [24, 25]. All show the inherent complexity of surface states even in the simplest 1D step array. The clear-cut case of the 1D QW in the $\mathrm{Au}(232321)$ surface is an exception, since surface states display a smoothly changing dimensionality from $1 \mathrm{D}$ to $2 \mathrm{D}$ within a wide range of $d$ values. Also exotic structure/electronic interferences arise due to Fermi surface "nesting" that occurs when the superlattice vector $2 \pi / d$ matches the Fermi wave vector $k_{F}[29,30,31]$, as shown in Fig. 7. Additionally, data display strong photon-energy dependent intensity variations, and fine features, such as gaps, are obscured by size distribution broadening. Yet photoemission spectra in vicinal surfaces always exhibit uniaxial anisotropy, with surface band changes in the direction perpendicular to the steps and flat surface behaviour in the parallel direction. In Fig. 8 we present a characteristic example of high resolution angle-resolved photoemission study in the sharply defined $(\sigma=0.1)$ step array of the $\mathrm{Au}(887)$ surface. The STM image indicates the presence of large (few micron size) homogeneous monatomic stepped areas, with well defined terrace size $(d=39 \pm 4 \AA)$. Across the step array, one 
can observe the umklapped $(2 \pi / d) N=1$ and $N=2$ mini-bands. These have been fitted with a periodic 1D Kronig-Penney model [23], rendering the strength of the repulsive barrier at the step edge $U_{0} b=1.8 \mathrm{eV} \times \AA$. Along the step array, high resolution permits to observe the free-electron-like dispersion $\left(m^{*}=0.25 m_{0}\right)$ of the $N=1$ band and its spin-orbit splitting, like in flat $\mathrm{Au}(111)[32,33,34]$.
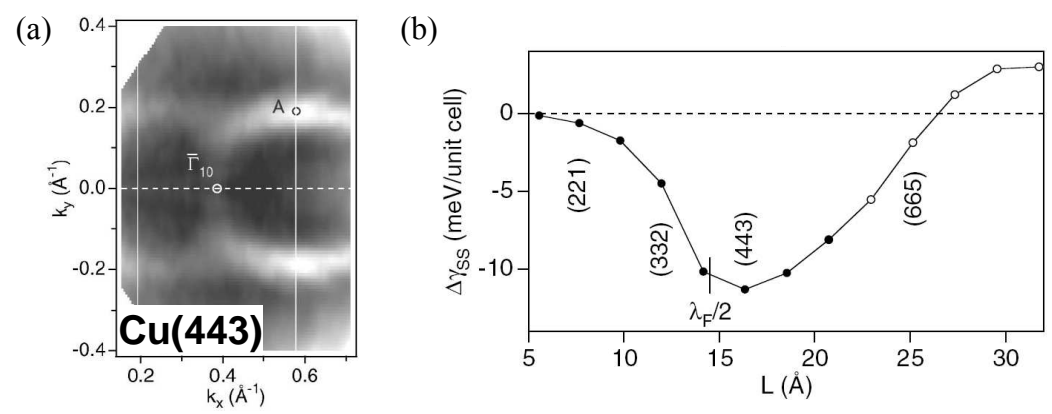

Fig. 7. (a) Fermi surface gap in the $\mathrm{Cu}(445)$ vicinal surface, where the terrace width $d=16 \AA$ makes it possible Fermi surface nesting $\left(\pi / d=k_{F}\right)$ in the direction perpendicular to the step array. (b) The surface tension change calculated for $\mathrm{Cu}(111)$ vicinals with superlatice bands shows a minimum around $d=16 \AA$, indicating that Fermi surface nesting conferes an extra stability to the $\mathrm{Cu}(443)$ plane (from [31]).

The complex nature and the repulsive character of the electron potential at steps is straightforwardly visualized by a direct comparison of high resolution photoemission spectra for flat and vicinal surface. The peaks in Fig. 9 (a) correspond to the bottom of the surface band for $\mathrm{Cu}\left(\begin{array}{lll}10 & 10 & 11\end{array}\right)\left(\begin{array}{l}d=43 \AA \\ \AA\end{array}\right)$ and $\mathrm{Cu}(111)$ surfaces, prepared and measured under the same experimental conditions [23]. The upwards energy shift $\Delta E=E-E_{0}=30 \mathrm{meV}$ and the extra broadening $\Delta W=W-W_{0}=35 \mathrm{meV}$ (FWHM) with respect to $\mathrm{Cu}(111)$ are the signatures of the scattering at steps in the $\mathrm{Cu}(1010$ 11) superlattice. Both peak shift and broadening increase as the density of steps (or miscut angle) increases, reflecting the repulsive nature of the step barrier and the local, step edge absorption (or leaking) into bulk states [35]. Moreover, the effective strength of the barrier potential $\left(U_{0} \times b\right)$ can be readily derived from $\Delta E$ using the simple 1D Kronig Penney model [24, 25], as sketched in Fig. 9 (b). The result of the fit in a variety of $\mathrm{Cu}(111)$ and $\mathrm{Au}(111)$ vicinals is shown in Fig. 9 (c). The barrier strength is reduced by an order of magnitude from a surface with relatively wide terraces $(\mathrm{Au}(232321), d=56 \AA)$ to surfaces with smaller step lattice constant $d \sim 20 \AA$, thereby making surface states smoothly evolve from quasi-1D QW-s in wide terraces to $2 \mathrm{D}$ surface bands in narrow terraces. This phenomenon, although not totally understood yet, appears to be connected with the evolution of the bulk projected band gap 
that supports the surface state, which progressively shrinks for surfaces with smaller $d$ values and vanishes around $d \sim 20 \AA[24,25]$.

(a)

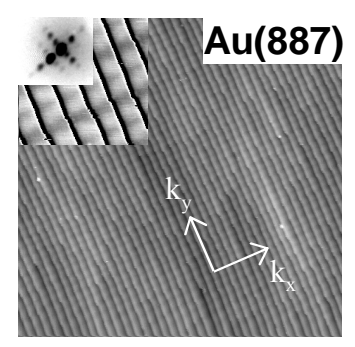

$150 \mathrm{~nm}$
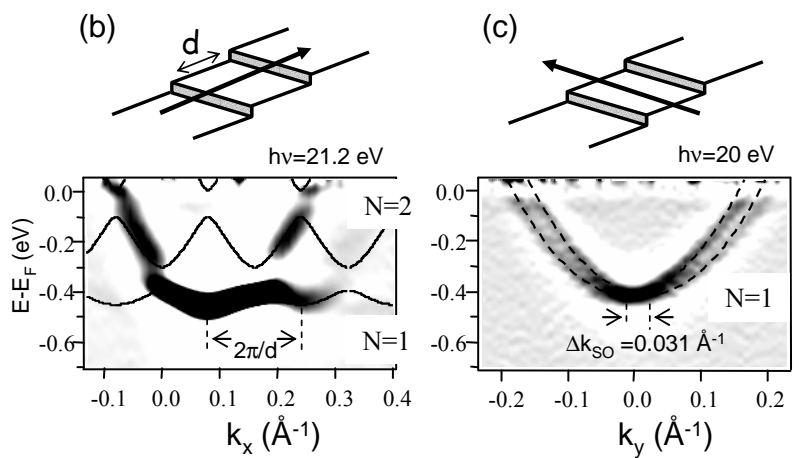

Fig. 8. High resolution photoemission experiments from the model, vicinal $\mathrm{Au}(887)$ surface. (a) STM topography showing the 1D step array. The periodic reconstruction pattern along the (111) terraces (inset) actually defines a square mesh, reflected in the fine electron diffraction pattern around the $(0,0)$ spot shown in the left corner. (b) $N=1$ and $N=2$ surface bands measured along $k_{x}$ and fitted with a $1 \mathrm{D}$ Kronig Penney model (lines). (c) Free-electron like dispersion along $k_{y}$, displaying the characteristic spin-orbit splitting of $\mathrm{Au}(111)$ surfaces [32, 33, 34] (adapted from $[24,23])$.

The terrace size distribution $(\sigma=<d>/ d)$ in vicinal surfaces gives rise to both energy level as well as wave vector broadening. The latter is related to the fact that surface bands are located at the surface Brillouin zone edge $(\pi / d)$ in the average surface plane. All in all, surface state peaks become broader in a vicinal surface due to both size distribution and lifetime effects. In Fig. 9 (d) we reproduce the recent peak width analysis of surface states collected from a variety of $1 \mathrm{D}$ stepped nanostructures. Independently of the system, a linear relationship between $\Delta E$ and $\Delta W$ is found [35]. The contribution from size distribution effects is minor (red line), and hence the linear relationship in Fig. 9 (d) indicates that lifetime broadening of surface states in vicinal surfaces depends on the step density $d$ and the barrier strength $U_{0} \times b$ in the same way as $\Delta E$. Thus local absorption at step edges appears to be the dominant source of inelastic scattering, supporting the model of complex step barrier potential proposed in Fig. 9 (b).

The uphill-downhill asymmetry that characterizes vicinal surfaces, as well as nanostructures grown on top, has been shown to affect surface state scattering through both the real and imaginary parts of the barrier potential $[35,36,37]$. This is the case of the $\mathrm{Ag} / \mathrm{Cu}$ nanostripe array shown in Fig. 10 (a) [35]. At 0.6 ML coverage, the STM picture (left) proves the presence of one monolayer thick $\mathrm{Ag}$ stripes that alternatively fill up terraces in the $\mathrm{Cu}(10$ 1011 ) substrate, as schematized on the top, right panel. Such particular mode 
(a)

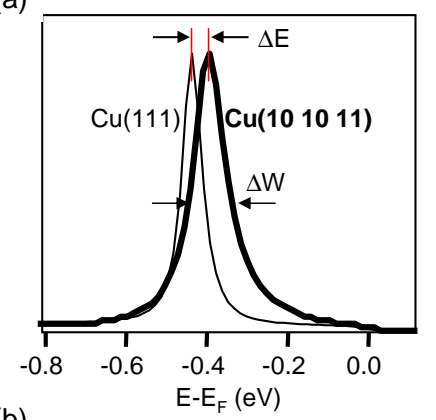

(b)

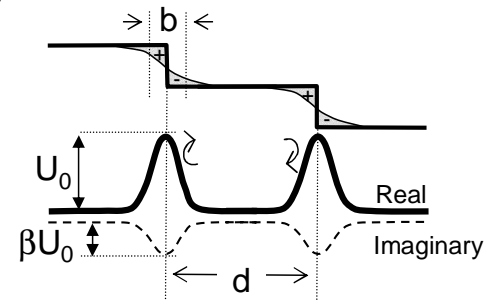

(c)

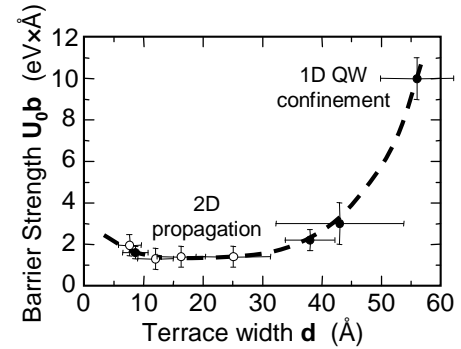

(d)

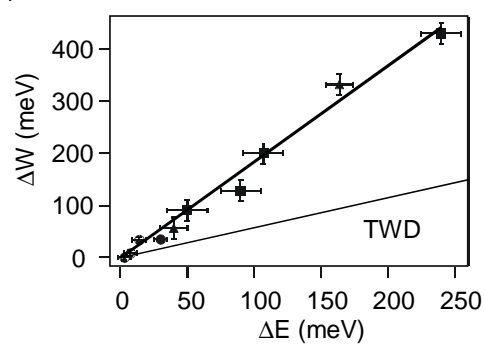

Fig. 9. (a) Surface state spectra of $\mathrm{Cu}(111)$ and $\mathrm{Cu}(101011)$ measured at band minima. The peak shifts $(\Delta E)$ and broadens $(\Delta W)$ in the vicinal surface, as expected for a step potential with real (repulsive) and imaginary (absorptive) parts, like the one sketched in (b). (c) The barrier strength $U_{0} \times b$ as determined from a number of stepped systems strongly varies as a function of the lattice constant $d$, such that surface states evolve from 1D QW-s at large $d$ values to $2 \mathrm{D}$ superlattice states with small $d$. (d) $\Delta E$ versus $\Delta W$ measured in a variety of stepped noble metal systems. The linear relationship proves a local step edge scattering scenario, supporting the complex barrier potential description in panel (b) (adapted from [23, 35]).

of growth is actually a consequence of the tendency to form large islands with triangular misfit dislocations on $\mathrm{Cu}(111)$, attached to surface steps [38]. The spectra on the bottom correspond to the surface state band minima compared to the flat system. One can observe step scattering, i.e., surface state shift and broadening, affecting electrons from Ag stripes (downhill scattering), but not electrons from $\mathrm{Cu}$ stripes (uphill scattering). The same asymmetric property is deduced in pump-probe experiments for image states on $\mathrm{Ag} / \mathrm{Pt}(997)$ nanostripes [37] and, as shown in Fig. 10 (b), in $\mathrm{Cu}(119)$ [36]. It is always found that the $N=2$ image state lifetime, which is the inverse of the decay rate plotted in Fig. 10 (b), is longer for electrons running uphill, in agreement with the $\mathrm{Ag} / \mathrm{Cu}$ scattering asymmetry observed in Fig. 10 (a). 
(a)
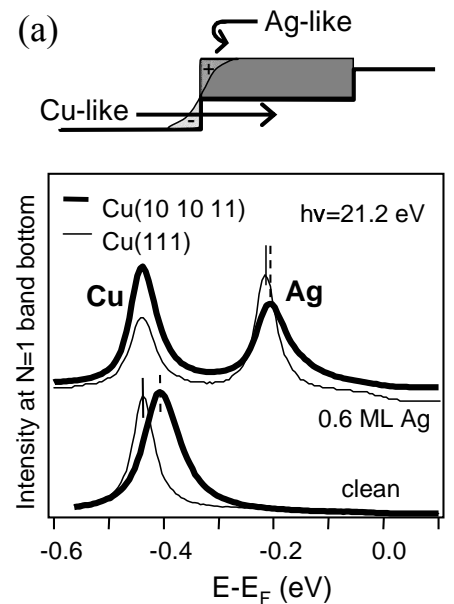

(b)
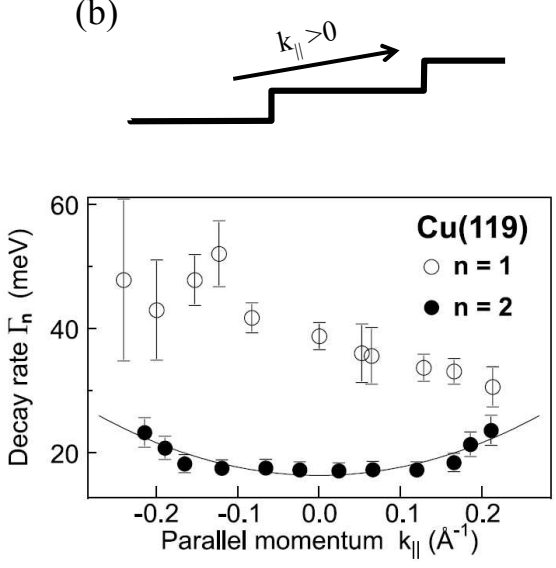

Fig. 10. Asymmetric scattering in stepped systems, proved in (a) surface states of $\mathrm{Ag} / \mathrm{Cu}$ nanostructures, where peak shift and broadening with respect to flat systems affect $\mathrm{Ag}$ states (downhill step scattering) but not $\mathrm{Cu}$ states (uphill step scattering), and (b) image states in $\mathrm{Cu}(119)$, where lifetime is longer for $N=1$ states running uphill (adapted from $[35,36]$ ).

\subsection{Exploring the 3D Fourier space: The modulation plane}

Surface state properties, such as energies or wave functions, are mostly determined by the bulk projected band gap supporting the surface state. For example, the size of the gap determines the effective penetration inside the bulk. Relatively large gaps lead to a few atomic-layer damping, whereas surface states in narrow gaps and surface-bulk resonances tail deep inside the crystal. In the vicinal surface the formal projection picture breaks, since due to umklapp with the step superlattice, projected band gaps would not exist. In reality, a "first-zone" projection scheme appears to hold [24, 25]. It has been proposed that the major changes observed in surface states of vicinal surfaces seem to be connected to the shrinking size of the first-zone projected band gap, i.e., to the direct overlap between surface states and bulk states and the subsequent formation of surface resonances [24, 25, 39]. In particular, the change in the effective strength of the barrier potential shown in Fig. 9 $[24,25]$. Indeed pure surface states have a high probability within the surface layer and hence feel a relatively strong step edge potential, by contrast to surface-bulk resonances that are located away from the surface plane, where the electrostatic dipole potential is smoothen out [40]. On the other hand, surface-bulk mixing via step lattice umklapps is always possible in a vicinal surface, but this would rather influence the surface state lifetime [36].

The changes in both the effective penetration of the surface state and the step barrier potential are reflected in a characteristic tilt of the wave function modulation plane, i.e., the direction along which its damping tail decays inside 
the bulk. That is determined by probing the Fourier spectrum in the direction perpendicular to the bulk $\left(k_{z}\right)$, as shown in Fig. 11. Such analysis, which requires photon-energy tuning and hence synchrotron radiation, is analogous to the $\left|\Psi_{N}\left(k_{x}\right)\right|^{2}$ mapping in the $k_{x}$ direction shown in Fig. 6. However, the quantitative $\left|\Psi_{N}\left(k_{z}\right)\right|^{2}$-mapping is limited by the short (2-3 layer) photoelectron escape depth that causes a large $k_{z}$ broadening, and hence only a qualitative analysis can be made [24, 25]. In Fig. 11 (a) we show the Fermi surface for $\mathrm{Cu}(335)$ measured at increasing photon energies. In Fig. 11 (b) we plot the corresponding $\left(k_{z}, k_{x}\right)$ values calculated at surface band minima, i.e., at the Fermi ring center. The analogous plot for the $N=1 \mathrm{QW}$ of $\mathrm{Au}(232321)$ is shown in Fig. 11 (c). In both cases, the continuous spectral distribution of the surface state is being discretely probed in the $k_{x}-k_{z}$ plane, allowing one to determine the modulation plane. One can observe a remarkable qualitative difference between $2 \mathrm{D}$ bands and 1D QW-s, i.e., data points line up along the average surface normal in $\mathrm{Cu}(335)$, by contrast to the [111] direction in $\mathrm{Au}(23$ 2321 ). This straightforwardly proves that the modulation plane switches from the average (optical) surface in $2 \mathrm{D}$ bands of $\mathrm{Cu}(335)$ to the (111) terrace in $\mathrm{Au}(232321)$.

(a)

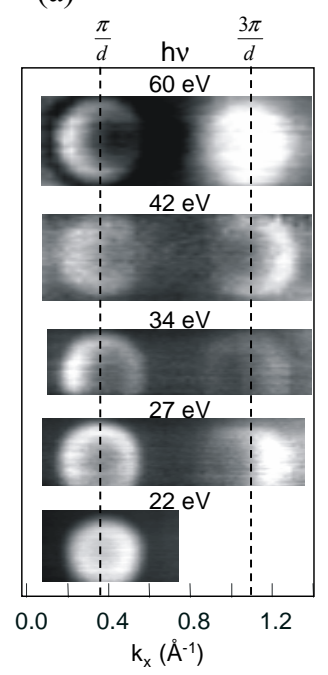

(b)

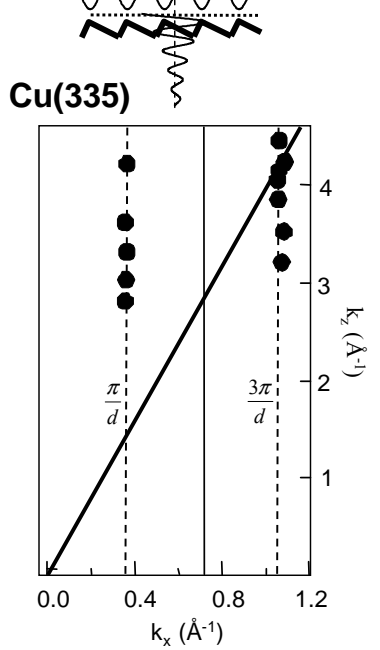

(c)
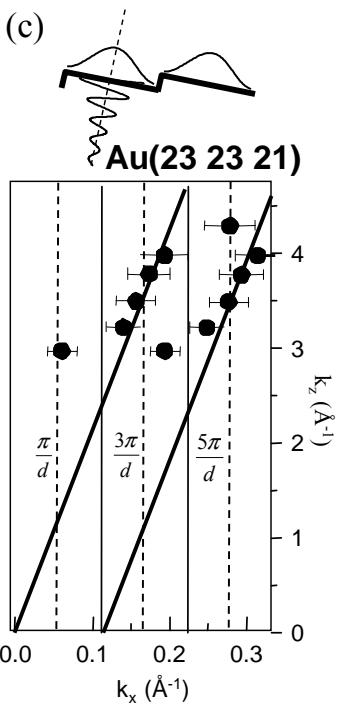

Fig. 11. Probing the modulation plane of surface states in vicinal surfaces by photon-energy-dependent photoemission. (a) Fermi surface rings for $\mathrm{Cu}(335)$ at increasing photon energies. (b) $\left(k_{z}, k_{x}\right)$ plot, where data points correspond to the surface band minimum (ring center) in (a). The two umklapped sets of points line up perpendicular to the average surface plane. (c) Analogous $\left(k_{z}, k_{x}\right)$ plot for the $N=1 \mathrm{QW}$ in $\mathrm{Au}(232321)$ [25]. In this case, data points are aligned along the [111] direction, indicating a tilt in the wave function modulation plane with respect to $\mathrm{Cu}(335)$, as sketched on top (from [25]). 
The effective proximity of the wave function to the outmost surface plane affects the modulation plane, as nicely proved for image states on the vicinal $\mathrm{Cu}(775)$ surface [40]. Figure 12 shows the band dispersion for the $N=1$ and the $N=2$ image states. The $N=2$ parabola displays the characteristic symmetric dispersion around $\bar{\Gamma}$ for a noble metal surface, by contrast to the $N=1$ band, which is asymmetric and its minimum is shifted away towards the [111] direction. Such distinct behavior is explained by the differnt distance of the respective wave functions to the surface plane, as shown on the right panel. The $N=2$ image state is located $\sim 12 \AA$ away from the surface, and hence it is being affected by a relatively smooth surface potential. By contrast, the $N=1$ state lies $\sim 3 \AA$ above the surface plane, making it more sensitive to the step corrugation.

(a)

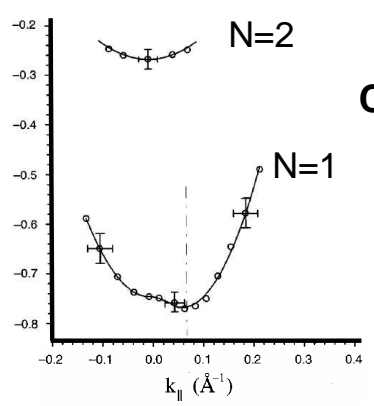

(b)

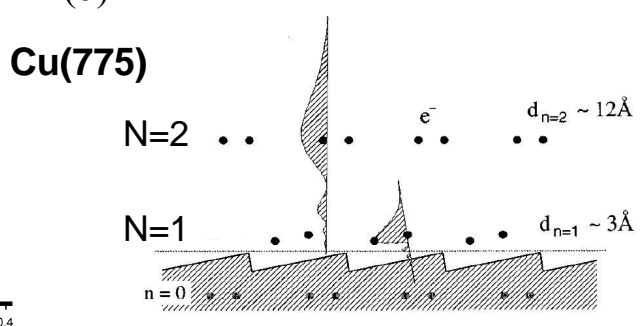

Fig. 12. Distinct modulation planes of image states in vicinal $\mathrm{Cu}(775)$, proved by the shift of the band with respect to the surface normal (left). $N=1$ and $N=2$ states lie close and away from the surface plane, respectively, defining the (111) terraces or the average surface as the modulation plane in each case (from [40]).

The analysis of surface states in step arrays and lateral nanostructures carried out during the past few years in a variety of systems has provided the basic ideas and the analytical framework necessary to understand electronic states in more complex systems using angular photoemission. It is clear that, beyond the spectrum of energy levels, one also needs to thorougly explore the three-dimensional Fourier space to probe, and eventually map, electron wave functions in real space. Such measurements will require high energy and angular resolution, as well as photon-energy tuning. In Fig. 13 is shown a good example, i.e., the extensive analysis of electronic states in the $\mathrm{Au}(11$ 9 9) surface [25]. This is a periodically faceted surface made of wide $d_{A}=42$ $\AA$ terraces and narrow $d_{B}=14 \AA$ step bunches. By selecting an appropriate photon energy $(17 \mathrm{eV})$ one is able of separating a sharp, non-dispersing $N=1$ peak from the broad, dispersing $N=2$ bands, as shown in Fig. 13 (a). In order to unveil the physical nature of both states, we examine the $\left(k_{x}-k_{z}\right)$ plot in Fig. 13 (b), and analyze the $k_{x}$-dependent photoemission intensity in Fig. 13 (c). The non dispersing peak leads to a single set of data points 
along the [111] direction in the $\left(k_{x}-k_{z}\right)$ plot, and to a probability density $\left|\Psi\left(k_{x}\right)\right|^{2}$ that fits to that of the $N=1$ state of the infinite QW of size $d_{A}$. Such behavior is indeed expected for a 1D QW mostly located inside terraces in Fig. 13 (d). On the other hand, the photon-energy analysis of the dispersing $N=2$ bands leads to five sets of vertical $2 \pi / D$ umklapps $\left(D=d_{A}+d_{B}\right)$ in the $\left(k_{x}-k_{z}\right)$ plot of Fig. 13 (b), with the photoemission intensity peaking at data sets separated by $\sim 2 \pi / d_{B}$. These are indeed the features for a $2 \mathrm{D}$ surface state strongly modulated within the step bunch, but propagating on the average surface plane along the $k_{x}$ direction, as shown in Fig. 13 (d).

(a)

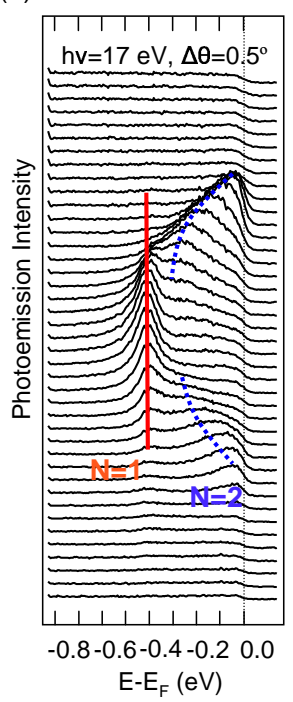

(b)

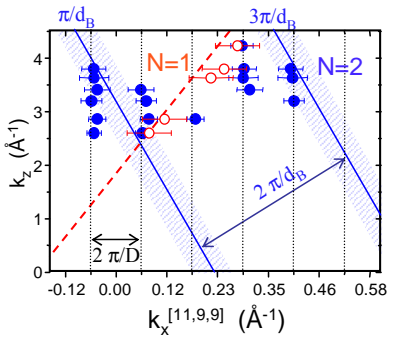

(c)

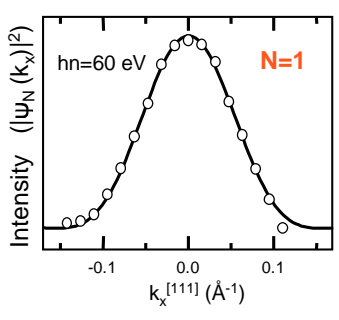

(d)

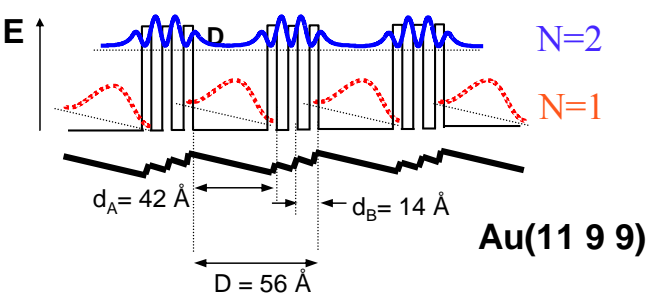

Fig. 13. Surface states in the faceted $A u\left(\begin{array}{lll}11 & 9 & 9\end{array}\right)$ surface, composed of terraces $\left(d_{A}\right)$ and periodic step bunches $\left(d_{B}\right)$, as shown in panel (d) [25]. (a) High resolution spectra showing distinct $1 \mathrm{D} N=1$ and $2 \mathrm{D} N=2$ features. (b) $\left(k_{x}-k_{z}\right)$ plot for the 2D (filled dots) and the 1D (open dots) features observed in (a). The data points for the $2 \mathrm{D}$ bands group forming five sets of $2 \pi / D\left(D=d_{A}+2 d_{B}\right)$ umklapps that line up perpendicular to the average surface, whereas the data for the non-dispersing feature follow the $4 \pi / D$ umklapp line of the (111) direction [25]. (c) Photoemission intensity as a function of $k_{x}\left(\left|\Psi\left(k_{x}\right)\right|^{2}\right)$ for the $1 \mathrm{D}$ state in (a) The line represents $\left|\Psi\left(k_{x}\right)\right|^{2}$ calculated for the $N=1$ state in the infinite QW of size $d_{A}=42 \AA$. (d) Wave function model deduced from (b) and (c) (from [25]). 


\section{Atomic Chains on Semiconductor Surfaces: The Ultimate Nanowires}

With the analysis tools for low-dimensional surface states in hand, we now proceed to the atomic chain structures that can be assembled at semiconductor surfaces. Their electronic structure is more complex than that of stepped metal surfaces, due to the localized broken bond orbitals of covalently-bonded semiconductors. On the other hand, the electronic states at a semiconductor surface de-couple completely from the bulk, as long as they reside in the bulk band gap. At these energies, surface states cannot hybridize with three-dimensional states. A variety of metallic semiconductor surfaces have been found in recent years, both two-dimensional [41, 42] and one-dimensional [43, 45, 12]. These combine the best of both worlds: The surface electrons are de-coupled from the substrate, but the surface atoms are locked into place by highly-directional, covalent back-bonds. Those are formed by three-dimensional orbitals with energies well below the Fermi level. The interesting states are those at the Fermi level, not the low-lying back-bond. States within a thermal energy of the Fermi level $\left(k_{B} T=25 \mathrm{meV}\right.$ at room temperature) are relevant to $2 \mathrm{D}$ and 1D transport, superconductivity, magnetism, charge density waves, and other more exotic phases predicted for 1D systems. The surface states can be tuned systematically from $2 \mathrm{D}$ to $1 \mathrm{D}$ by varying the spacing between atomic chains via the step spacing. Moving the chains farther apart reduces the coupling exponentially with a decay constant of atomic dimensions. As a result, the $1 \mathrm{D} / 2 \mathrm{D}$ coupling ratio can be varied from $12: 1$ to $>70: 1$. At present, the detection limit of 70:1 is imposed by the finite angular resolution of photoelectron spectrometers $[?, ?]$.

\subsection{Self-Assembly of Atom Chains on Stepped $\operatorname{Si}(111) 7 \times 7$}

The construction of atomic chain structures on vicinal $\mathrm{Si}(111)$ surfaces $[15,47$, 58, 49] is illustrated in Figs. 14 and 15. The clean surface exhibits a regular array of facets, which are stabilized by the deep $7 \times 7$ reconstruction and multiple steps. The data are from the $\mathrm{Si}(557)$-Au structure, which consists of one gold chain every five atomic rows. It is among the best-studied 1D surfaces, and will serve as prototype for demonstrating the phenomena encountered in atomic chains on semiconductors. Another well-studied surface is $\mathrm{Si}(111)$ $4 \times 1$-In [43, 44], where four indium chains per unit cell form two closely-spaced zig-zag rows. The four chains produce three metallic bands that interact with each other. This surface serves as an example for the richness of the phenomena occurring in atomic chains. Chain structures can be obtained with a large variety of metal atoms, such as alkalis, alkaline earths, noble metals ( $\mathrm{Ag}, \mathrm{Au})$, transition metals $(\mathrm{Pt})$, and rare earths. Rare earth chains bring $f$-electrons with large magnetic moments into the picture and thus become prototypes for spin chains. All rare earth chain structures studied so far on $\mathrm{Si}(111)$ exhibit a similar $5 \times 2$ structure, consisting of alternating chains of filled and empty 

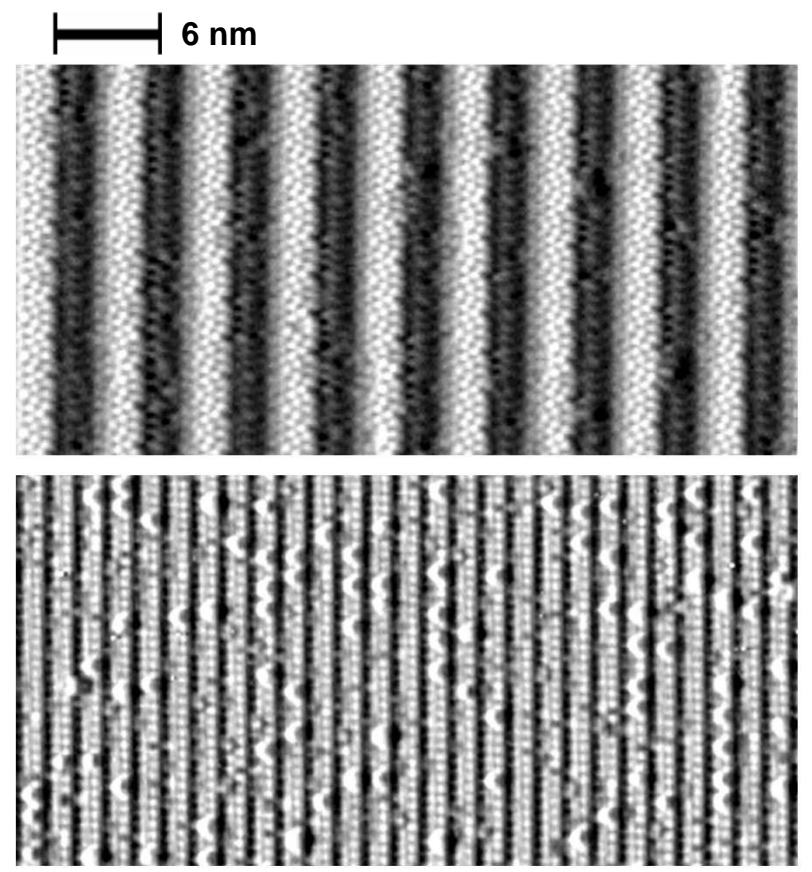

Fig. 14. Fabrication of atomic chains on a stepped $\operatorname{Si}(111)$ surface. The clean $\operatorname{Si}(557)$ surface forms regular facets that contain one $7 \times 7$ unit cell and a triple step (top). Deposition of $1 / 5$ of a monolayer of Au removes the facets and creates atomic chains. The two chains seen by STM are Si atoms with broken bonds, not gold atoms. This and all following STM images display the derivative of the height in the direction perpendicular to the steps. This gives the appearance of a surface illuminated at grazing incidence, with steps casting dark shadows. From [46],[77],[74].

orbitals [15]. That provides the opportunity of systematically varying the spin by changing the $f$-count while keeping the structure the same.

Not all chain structures are metallic, but most of the Au-induced chains are. Essentially, gold forms a metallic chain structure on every vicinal $\mathrm{Si}(111)$ surface with odd Miller indices that has been studied. They consist of two classes with steps going uphill and downhill, as shown in Fig. 14. With a tilt of the surface normal towards $[\overline{1} 12]$ there are two broken bonds at the step edge of the truncated bulk structure (such as (335), (337), (557)). With the opposite tilt towards $[11 \overline{2}]$ there is only one broken bond at the step edge (such as (110), (553), (775), (995), (13 13 7) [12]). Even on the flat $\operatorname{Si}(111)$ surface, the three-fold symmetry is broken by gold and other metals. Three domains of a chain structure are formed. A single domain with chains parallel to the steps can be selected by a shallow miscut towards [1112]. The most prominent example is $\mathrm{Si}(111) 5 \times 2$-Au with two gold chains in five atom rows ([50, 56, 52] and references therein). 


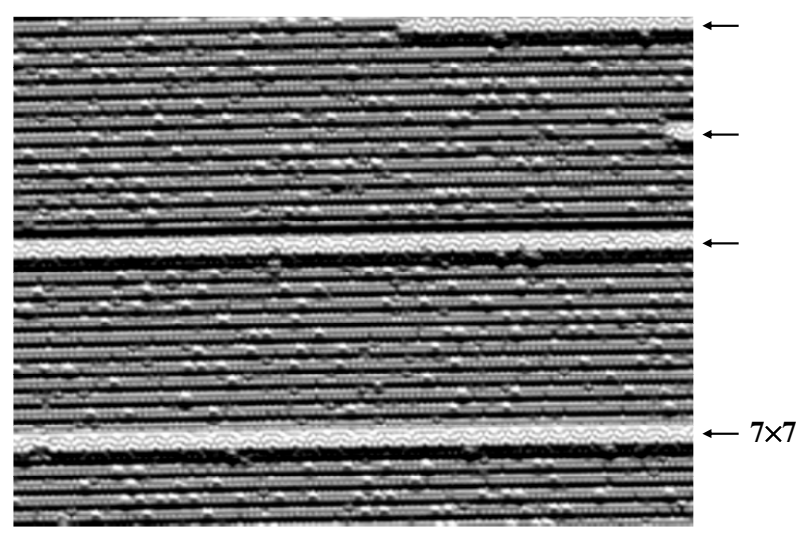

Fig. 15. One-dimensional growth pattern of the Si(557)-Au chain structure. Long strips of clean $\operatorname{Si}(111) 7 \times 7$ remain, when the gold coverage is slightly below the optimum (by $2 / 100$ of a monolayer). These are a single $7 \times 7$ cell wide. From [10].

The one-dimensional character of these surfaces can be seen already during growth, as shown in Fig. 15. At a slight under-coverage (only 2/100 of a monolayer below the optimum of $1 / 5$ monolayer), one finds long strips of clean $\operatorname{Si}(111) 7 \times 7$ that have not been converted yet. These are exactly one $7 \times 7$ unit cell wide. Two $7 \times 7$ strips on the left side are in the process of being consumed by $\mathrm{Au}$ chains. This explains why an accurate $\mathrm{Au}$ coverage is the single most important criterion for preparing high quality chain structures. The chains extend for long distances if the azimuthal orientation of the vicinal surface is correct (compare [53,54] for the optimum preparation condition of stepped $\mathrm{Si}(111)$ surfaces). If the orientation is slightly off, the resulting kinks can be swept together into bunches by electromigration with a DC heating current parallel to the steps. Since this effect is uni-directional, it is advisable to try both directions in order to sweep out both left- and right-handed kinks. Kink-free chains have been achieved over distances comparable to the scanning range of a STM $(\sim 1 \mu \mathrm{m})$, with up to 20000 edge atoms between kinks. The least-critical parameters are the substrate temperature during Au deposition $\left(600-700^{\circ} \mathrm{C}\right)$ and the subsequent post-anneal $\left(800-900^{\circ} \mathrm{C}\right.$ for a few seconds).

\subsection{Atomic Structure}

The atomic structure of these surfaces has been remarkably difficult to pinpoint. The best-studied surfaces are $\operatorname{Si}(557)-\mathrm{Au}$ and $\mathrm{Si}(111) 5 \times 2-\mathrm{Au}$, which have been investigated by surface x-ray diffraction, electron diffraction, and local density calculations with total energy minimization and modeling of the STM topography $[55,56]$. An increasing number of low-energy structures is being discovered in thorough theoretical searches [57]. The task is made difficult by the extra degrees of freedom introduced at a step edge, where additional 
silicon atoms can attach themselves rather easily. In fact, such silicon adatoms frequently serve as dopants that optimize the band filling of the chains [56].

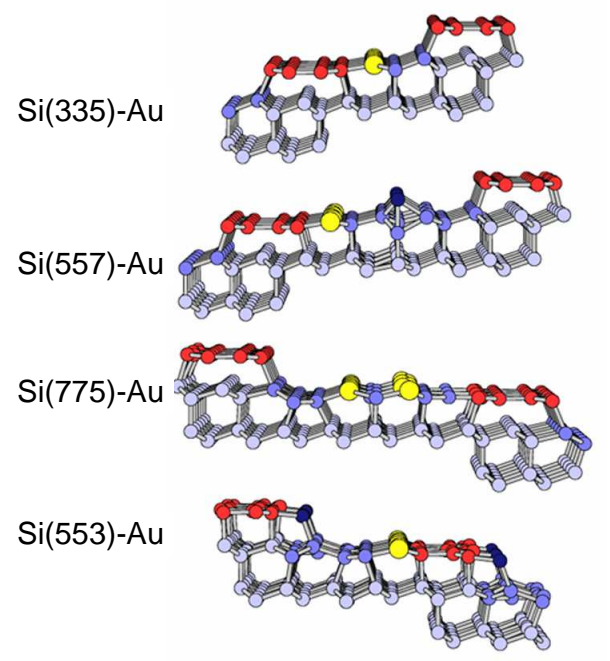

Fig. 16. A few of the chain structures induced by $\mathrm{Au}$ on vicinal $\mathrm{Si}(111)$, obtained from total energy minimization. They have two structural elements in common: 1) A honeycomb chain of graphitic silicon, which drives the surface one-dimensional (red). 2) A chain of gold atoms at the center of the terrace, contrary to a simple model of step flow growth (yellow). The terrace width can be varied in increments of two row spacings, which allows tailoring of the inter-chain coupling. From [12].

With a certain caveat, one can isolate two features that are common to many $1 \mathrm{D}$ structures on $\mathrm{Si}(111)$, including those induced by gold, alkali metals, and alkaline earths. These are illustrated with the results of total energy calculations for a few Au-induced chain structures in Fig. 16.

1) A common structural element is the honeycomb chain [58], which consists of a graphitic strip of Si hexagons. $\pi$-bonding is unexpected for silicon, but many silicon surfaces exhibit $\pi$-bonding in one form or another, such as dimmers on $\mathrm{Si}(100)$ and polyacetylene-like chains on cleaved $\mathrm{Si}(111)$. Broken bonds go through unusual contortions in order to regain some bonding energy. The honeycomb chains are highly one-dimensional. They can be extremely long (hundreds of nanometers), but they are less than two hexagons wide. The lattice match must be nearly perfect along the graphitic stripe (in the [11̄0] direction), but very poor perpendicular to it (along [112]). From these observations it is reasonable to conjecture that the honeycomb chains are the driving force that stabilizes $1 \mathrm{D}$ surface structures over potential $2 \mathrm{D}$ competitors, which are typically $\sqrt{3} \times \sqrt{3}$ structures. $2 \mathrm{D}$ structures does not appear until full monolayer coverage is reached. 
2) The second structural element is the metal chain. Judging from semiconductor homoepitaxy, one might expect the metal atoms to go to the step edge, as in step-flow growth. After all, there are extra bonding possibilities to the upper step edge. The gold chain structures defy such simple ideas and place themselves right in the middle of a terrace (Fig. 16). Instead of sitting on top, the Au chain is firmly incorporated into the $\mathrm{Si}(111)$ surface by substituting for a $\mathrm{Si}$ surface atom. For $\mathrm{Si}(557)-\mathrm{Au}$, this geometry is confirmed by both x-ray diffraction [59] and total energy calculations [12]. STM is not able to see the $\mathrm{Au}$ chain, because each $\mathrm{Au}$ atom combines its $s, p$-electron with a Si broken bond and forms a filled band about 1-2 eV below $E_{F}[60,55]$. Such a bound state has too little electron density outside the surface to be seen by STM.

The two atomic chains observed by STM on Si(557)-Au in Figs. 14, 15 actually originate from Si atoms with broken bonds, not from gold - another counter-intuitive feature of these 1D structures. One of them (the betterresolved chains on the right) can be assigned to the Si adatoms in the surface structure (dark blue in Fig. 16), the other to the step edge at the border of the honeycomb chain. This observation nay be generalized to chain structures formed by other metals, such as alkali metals and alkaline earths: The metal seems to play the role of a catalyst which facilitating the formation of the silicon honeycomb chain.

\subsection{Metallic Surface States in 2D}

The focus of this section will be on metallic surfaces, which have sharp states at the Fermi level $E_{F}$ that are de-coupled from the 3D bulk. Before discussing one-dimensional chain structures, it is useful to consider 2D surface states. They show already the importance of surface dopants in low-dimensional structures, which becomes even more important in 1D. The natural examples are the $\sqrt{3} \times \sqrt{3}$ structure of $\mathrm{Au}$ and $\mathrm{Ag}$ on $\mathrm{Si}(111)$, which displace chain structures when the coverage comes close to a monolayer [42, 41]. Simplified structural models are shown in Fig. 17.

An exactly stoichiometric $\sqrt{3} \times \sqrt{3}$ structure with 1 monolayer coverage is actually semiconducting, with a gap significantly smaller than the bulk band gap of $\mathrm{Si}[61,62,63]$. However, extra metal atoms deposited on this surface beyond a monolayer dope the surface conduction band and create a metallic surface (large circles in Fig. 17). The metallicity of the surface becomes visible in high-resolution photoemission after adding as little as 1.5/1000 of a monolayer (Fig. 18). With increasing metal coverage the radius of the Fermi circle increases, enclosing the extra electrons in the conduction band. The surface conduction band becomes visible as it fills up. However, it does not follow a simple rigid band model. Instead of remaining fixed with respect to the bulk valence band maximum (VBM, tickmarks on the side), the bottom of the surface band drops in energy with increasing doping. It starts out above the VBM, crosses the VBM at about 1/100 of a monolayer and drops several 


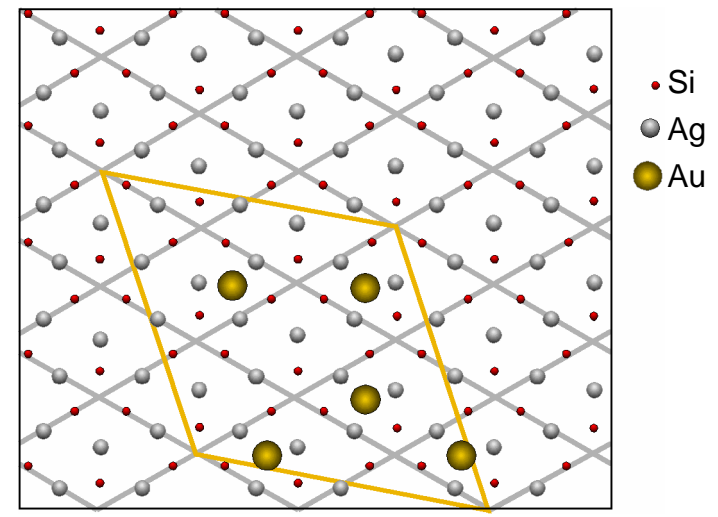

Fig. 17. Simplified model of two-dimensional metallic structures formed by $\mathrm{Ag}$ and $\mathrm{Au}$ on $\mathrm{Si}(111)$ at a coverage of 1 monolayer and above (from [16]). One-dimensional structures are typically formed at $1 / 5$ of a monolayer.

tenths of an eV below the VBM at the highest doping levels. The maximum achievable doping is reached with a $\sqrt{21} \times \sqrt{21}$ superstructure on top of the $\sqrt{3} \times \sqrt{3}$ superlattice, as shown in Fig. 17. This structure has a band filling of $5 / 21 \approx 0.24$ electrons per $1 \times 1$ unit cell.

The Fermi surface of the $\sqrt{21} \times \sqrt{21}$ "super-duper" structure is shown at the center of Fig. 18, together with the lightly-doped $\sqrt{3} \times \sqrt{3}$ structure on top [16]. Both exhibit Fermi circles centered at the smallest reciprocal lattice vectors of the $\sqrt{3} \times \sqrt{3}$ lattice. Surprisingly, the equivalent circle at the center of the images $(\mathbf{k}=0)$ is absent for the $\sqrt{3} \times \sqrt{3}$ structure and very faint for the $\sqrt{21} \times \sqrt{21}$ structure. This could be due to strong polarization selection rules or to the proximity of bulk Si states at the VBM near $\mathbf{k}=0$. An additional feature of the $\sqrt{21} \times \sqrt{21}$ structure is the intricate network of weaker Fermi circles. These can be explained rather simply as replicas of the dominant circle, shifted by the reciprocal lattice vectors of the $\sqrt{21} \times \sqrt{21}$ structure (see [16], Fig. 18). Furthermore, the corresponding energy bands exhibit mini-gaps at their intersections, whose magnitude is twice the superlattice potential acting on the surface electrons. The resulting potential is $55 \mathrm{meV}$ in this case [16], a good example of a small energy scale at a surface requiring high resolution (both in energy and angle).

A quite different, but very prominent metallic semiconductor surface is $\mathrm{Si}(111) 7 \times 7$ itself, the most stable silicon surface. The large unit cell leads to a very small Brillouin zone, which pushes the angular resolution capabilities of current spectrometers. Nevertheless, it has been possible to resolve its Fermi surface [64]. The electronic structure within a few meV of $E_{F}$ is still largely unknown, but there have been intriguing hints about a possible small Hubbard gap with a Kondo peak at the center from local density calculations augmented by Coulomb interaction terms [65, 66]. A Hubbard gap has 


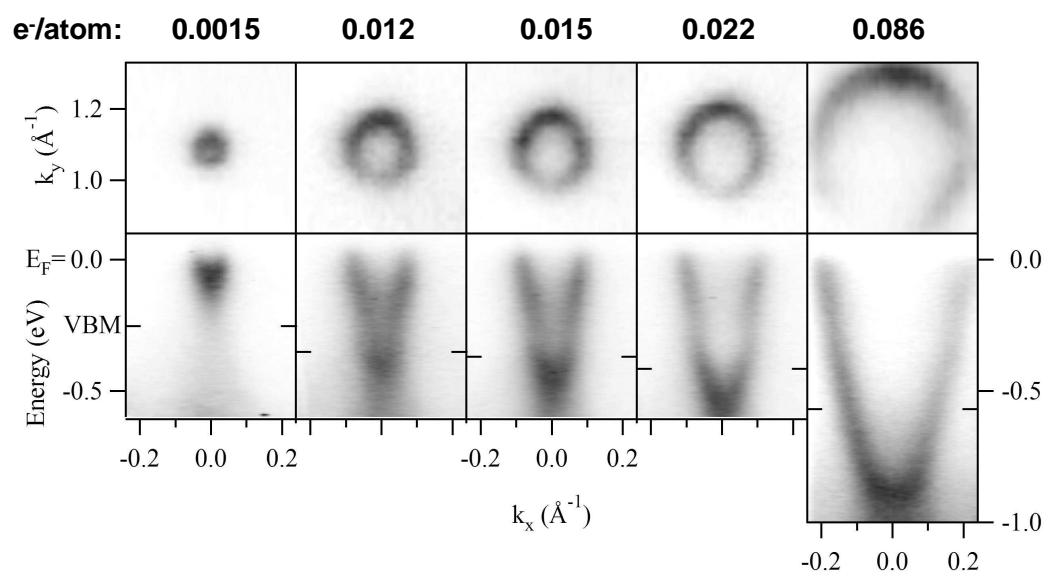

Fig. 18. Fermi surfaces and band dispersions for the two-dimensional structures on $\mathrm{Si}(111)$ shown in Fig. 17. A surface state band can be doped continuously by excess $\mathrm{Ag}$ or $\mathrm{Au}$ atoms on top of the stoichiometric $\mathrm{Si}(111) \sqrt{3} \times \sqrt{3}-\mathrm{Ag}$ structure (from [42]). The analogous plot for one-dimensional structures is given in Fig. 1. One-dimensional chains are doped by excess Si atoms.

been inferred as well from electron energy loss spectroscopy [67] and surface NMR measurements of the carrier density [68]. However the magnitude of the proposed Hubbard gap varies by an order of magnitude. Currently, the $7 \times 7$ surface is proving itself as ideal testing ground for electron-phonon interaction at semiconductor surfaces. The spectral function near $E_{F}$ can be described remarkably well by a combination of a bare and a phonon-dressed bands with an electron-phonon coupling parameter $\lambda=1.1$ [69]. There is a well-defined phonon mode at $70 \mathrm{meV}$ from a vibration of a $\mathrm{Si}$ adatom against the atom underneath. It interacts with the band at $E_{F}$, which is derived from states localized on the adatom as well.

\subsection{Electronic States from $2 \mathrm{D}$ to $1 \mathrm{D}$}

The topology of the Fermi surface changes dramatically between 2D and 1D, as demonstrated in Section 1.3 and Fig. 2. Two-dimensional Fermi surfaces are characterized by closed loops, such as the Fermi circles observed for the $\mathrm{Si}(111) \sqrt{3} \times \sqrt{3}-\mathrm{Ag}$ and $\operatorname{Si}(111) \sqrt{21} \times \sqrt{21}-(\mathrm{Ag}+\mathrm{Au})$ structures. A truly onedimensional Fermi surface consists of two points at $\pm k_{F}$, but these become straight lines when plotted in two dimensions. The energy is independent of the momentum perpendicular to the chains (along the $y$-axis in Fig. 2). An actual chain structure, such as $\mathrm{Si}(553)$-Au in Fig. 20, displays undulating lines. The amplitude of the undulations is a measure of the residual two-dimensional coupling. The complete data set can be reproduced by a simple tight binding calculation involving coupling energies to three sets of neighbor atoms, $t_{1}$ and $t_{3}$ for first and second neighbor along the chain and $t_{2}$ one between chains 
(see Fig. 19 left and [11]). The dimensionality ratio is given by $t_{1} / t_{2}$. For this particular structure one observes a doublet of closely-spaced Fermi lines with $t_{1} / t_{2}=39,46$ and a single line with $t_{1} / t_{2}=12$. The $\operatorname{Si}(553)$-Au surface has a fairly close chain spacing of $1.48 \mathrm{~nm}$. By going to the $\mathrm{Si}(557)$-Au surface with a somewhat larger chain spacing of $1.92 \mathrm{~nm}$, the $1 \mathrm{D} / 2 \mathrm{D}$ coupling ratio becomes so small that it cannot be measured any more ( $>70: 1)$. The decay constant for the $2 \mathrm{D}$ coupling is so short, that the $1 \mathrm{D}$ limit can be approached rather rapidly. The absolute value of the dominant $1 \mathrm{D}$ coupling $t_{1}$ is about $0.7 \mathrm{eV}$ for the doublet band that appears in both structures [12]. The effective mass is typically $1 / 2$ in these chain structures, and the Fermi velocity about $1 \cdot 10^{6} \mathrm{~m} / \mathrm{s}$ [11], which makes them rather free-electron-like along the chains.

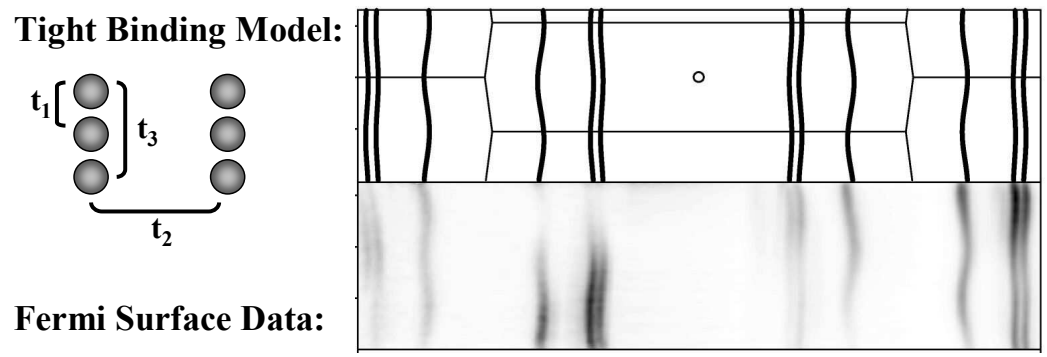

Fig. 19. Obtaining the $1 \mathrm{D}$ versus $2 \mathrm{D}$ coupling ratio from a tight binding fit to the Fermi surface of atomic chain structures, demonstrated for $\mathrm{Si}(553)-\mathrm{Au}$. The undulations in the Fermi lines are due to $2 \mathrm{D}$ coupling between the chains (from [11]). The $1 \mathrm{D} / 2 \mathrm{D}$ coupling ratio can be varied from 10:1 to >70:1 in Au-induced chain structures belonging to the family shown in Fig. 16

In order to follow the transition from $2 \mathrm{D}$ to $1 \mathrm{D}$, it is not even necessary to go from one structure to another with different chain spacing. The same surface can have bands with different dimensionality crossing the Fermi level, as demonstrated with the $\mathrm{Si}(553)-\mathrm{Au}$ surface and with $\mathrm{Si}(111) 4 \times 1-\mathrm{In}$ in Section 3.8. And the $\operatorname{Si}(111) 5 \times 2$-Au surface even contains a single band that changes its dimensionality from 1D at the top to 2D at the bottom [77]. Experiment and theory agree quantitatively on the change in the $1 \mathrm{D} / 2 \mathrm{D}$ coupling ratio [56].

The game of gradually reducing dimensionality can be pursued further from 1D to $0 \mathrm{D}$ by breaking up the chains into sections with adsorbates. A zero-dimensional surface state has been found at the end of a finite chain on $\mathrm{Si}(553)-\mathrm{Au}$ by scanning tunneling spectroscopy [70]. The atoms at the end of a finite chain appears either bright or dark, depending on the bias voltage used for the spectroscopic imaging. This phenomenon can be modeled by a simple calculation, thus making it a textbook example of quantum mechanics in very low dimensions. The $\mathrm{Si}(553)$-Au surface is particularly useful for this purpose because it is the most perfect of the Au-induced chain structures studied so 


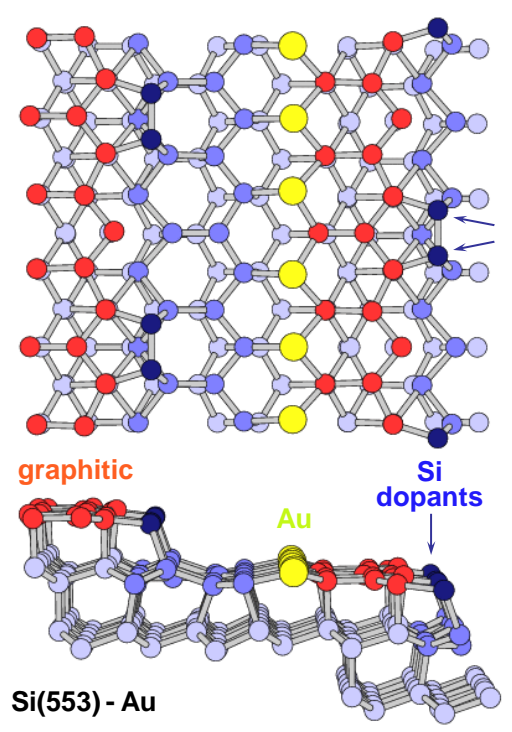

Fig. 20. Structural model of the Si(553)-Au surface from total energy minimization (from [12]). Extra Si atoms at the step edge dope the Au chain at the center of the terrace and lead to fractional band filling

far. The length of its chains can be as long as 100 atoms and chains with finite length can be selected over a wide range [71].

\subsection{Fractional Band Filling}

In addition to the $1 \mathrm{D} / 2 \mathrm{D}$ coupling ratio, the band filling is an important quantity determining the position of these electronic states in universal diagrams of $1 \mathrm{D}$ phases [6]. The filling of a band can be determined from the occupied area between Fermi surfaces and normalizing it to the Brillouin zone [12]. The latter is indicated in Fig. 19, with the circle near the center defining the $\Gamma$-point at $\mathbf{k}=0$. Since the bands are free-electron-like, Luttinger's theorem tells us that each band holds two electrons per unit cell (one for spin up and one for spin down). Thus, the area of the Brillouin zone corresponds to two electrons per atom. Each of the two closely-spaced bands occurring in most $\mathrm{Au}$ chain structures is about half-filled and thus contains one electron if unpolarized (1/2 electron if spin-split, as in a recent model [?]). The surfaces tilted towards the $[11 \overline{2}]$ azimuth (lower half of Fig. 16) contain an extra single band with a filling of about $1 / 3$, i.e. $2 / 3$ of an electron per unit cell. Together with the half-filled doublet the filling comes out to be very close to $8 / 3$ of an electron per unit cell ( $5 / 3$ for a spin-split doublet).

The fractional band filling of some of the gold chain structures raises interesting questions about possible connections to the fractional quantum Hall effect in 2D. However, the photoemission measurements are performed without 
a magnetic field, and even with an applied field one might question whether the analog of a two-dimensional Landau orbit exists for a one-dimensional chain. There is a less exotic, but nevertheless intriguing explanation the fractional filling. The $\mathrm{Si}(553)-\mathrm{Au}$ surface exhibits a tripling of the unit cell near defects $[11,70,71,72,73]$. In fact, the structural model with the lowest total energy (among about 50 models tested [12]) has $3 \times 1$ periodicity along the chains, as shown in Fig. 20. Two extra Si atoms are incorporated at the step edge per $3 \times 1$ unit cell. The Au chain at the center of the terrace, on the other hand, does not see much of the $3 \times 1$ periodicity at the step edge. It exhibits $1 \times 1$ periodicity in STM at room temperature and distorts into $3 \times 1$ only near defects and at low temperature [72,73]. Judging from the role of extra $\mathrm{Si}$ atoms as dopants (discussed below), it is likely that the extra Si atoms dope the $\mathrm{Au}$ chains. If the two $\mathrm{Si}$ atoms donated all their 8 valence electrons, that would make $8 / 3$ of an electron per Au atom. This is the upper limit. It is more likely that only two of the four $\mathrm{Si}$ valence electrons dope the chains, while the other two electrons are needed for covalent bonds (compare the calculations for $\mathrm{Si}(111) 5 \times 2-\mathrm{Au}[56]$ discussed below). In that case, one would have an electron count of $4 / 3$ of an electron per Au atom, which is close to the $5 / 3$ expected in a scenario with spin-split bands. In any case, a fractional filling seems to be closely tied to the existence of extra $\mathrm{Si}$ atoms at the step edge that act as dopants for the gold chains in the middle of the terrace. This type of indirect doping allows for high mobility of electrons near the Au chains, which are not disturbed by dopant atoms. The 1D Au chains are very much the $1 \mathrm{D}$ analog of the $2 \mathrm{D} \mathrm{CuO}$ planes in high temperature superconductors. In both cases the dopants reside in the next higher dimension.

\subsection{Doping of Chains and Nanoscale Phase Separation}

The observation of fractional band filling suggests that doping of 1D gold chains is rather peculiar. They cannot be doped continuously like their twodimensional counterparts in Section 3.3. Each of them has a well-defined optimum doping, which can be shifted only by a few percent with excess Au or $\mathrm{Si}$. The role of dopant atoms has been investigated closely for the $\operatorname{Si}(111) 5 \times 2$ $\mathrm{Au}$ structure, which is shown in Fig. 21. On top of faint atom chains with $5 \times 2$ periodicity one finds additional atoms in regular $5 \times 4$ lattice sites. However, the extra atoms occupy only half of the available $5 \times 4$ sites. They do this in seemingly random fashion, not by forming the regular $5 \times 8$ lattice expected at that coverage. Closer inspection reveals that the extra atoms form a complete $5 \times 4$ lattice for typically five sites, followed by an empty stretch of comparable length. This behavior can be quantified by determining the pair correlation function for STM images with thousands of atoms, and the result can be modeled by a nearest neighbor repulsion together with an oscillatory potential with $5 \times 4$ periodicity [76]. These interatomic potentials are in the meV regime, and they correspond to mini-gaps in the Fermi surface of the order 10-100 meV when assuming an electronic origin. The additional atoms can be identified 


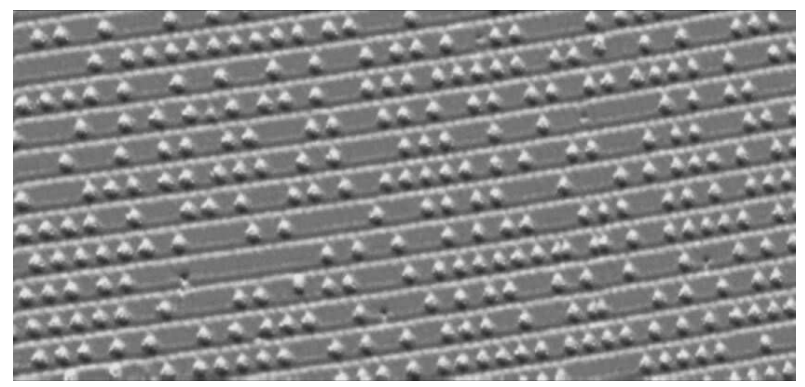

Fig. 21. Si dopants adsorbed on top of the chains of the $\mathrm{Si}(111) 5 \times 2$-Au structure, forming sections of a filled $5 \times 4$ lattice alternating with empty $5 \times 2$ sections (from [74]). The filled (doped) sections are semiconducting, and the empty sections metallic (see Fig. 22). This is a one-dimensional analog of nano-scale phase separation ("stripes") observed in high temperature superconductors. This structure can be used to fabricate an atomic scale memory, where a bit is stored by the presence or absence of a single Si atom [75].

as single Si atoms, not the deposited Au atoms: Deposition of an extra 1/40 of a monolayer of $\mathrm{Si}$ and annealing below $300^{\circ}$ creates a metastable structure where all $5 \times 4$ sites are filled [75].

Particularly interesting is the electronic structure of these alternating segments of filled and empty $5 \times 4$ sites. As shown in Fig. 22, the empty segments are metallic, while the filled segments are semiconducting with a $0.5 \mathrm{eV}$ gap and $E_{F}$ located near the bottom of the gap [13]. This behavior is reminiscent of the "stripes" in high temperature superconductors, where metallic, doped regions alternate with gapped, undoped regions on a nanometer length scale. These static stripes, and their dynamical counterparts are viewed as a special mechanism of bringing two incompatible ingredients of high temperature superconductivity together, magnetism and superconductivity. The segments of doped and undoped Si chains may be viewed as one-dimensional analog of stripes. The only difference is that metallic segments are undoped, and vice versa. Thus, the $\mathrm{Si}(111) 5 \times 2$-Au surface may serve as prototype for the formation of stripes in general.

For 1D chains there is a model explaining the formation of doped and undoped segments [52], and it is again a compromise between two conflicting requirements. The $\mathrm{Si}(111) 5 \times 2-\mathrm{Au}$ structure has an optimum doping of one $\mathrm{Si}$ atom per $5 \times 8$ cell according to total energy calculations [56]. However, a $5 \times 4$ surface periodicity is dictated by the Fermi surface measured with angleresolved photoemission [52]. It consists of ideally-nested Fermi lines located at the zone boundaries of the $5 \times 4$ lattice. A $5 \times 4$ reconstruction opens a minigap at these points, which reduces the energy of the occupied states. This is similar to a Peierls gap, except for a quadrupling of the period instead of a doubling. The $5 \times 4$ periodicity of the inter-atomic potential [76] has probably 


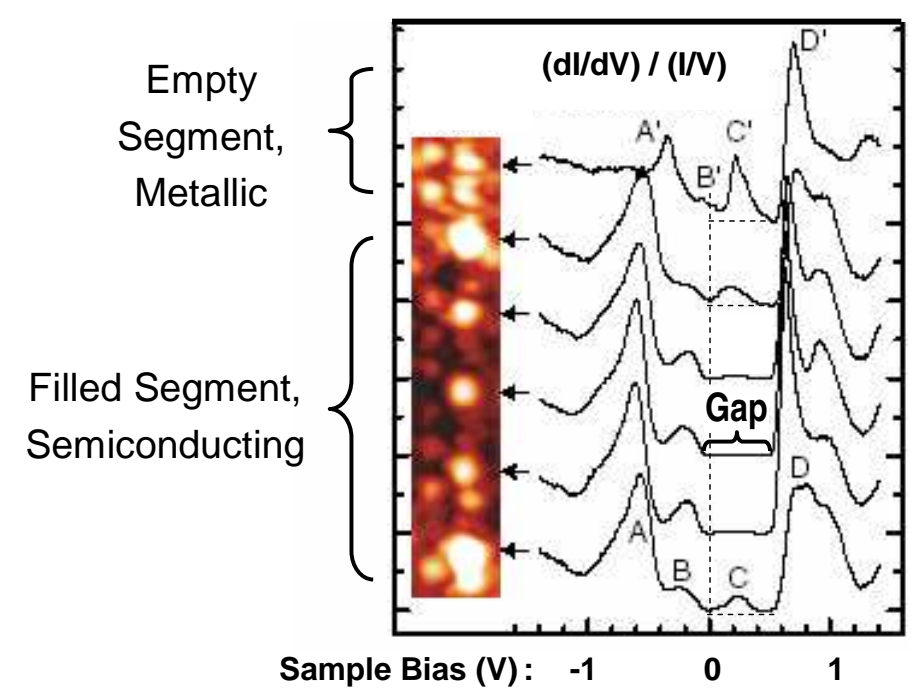

Fig. 22. Scanning tunneling spectroscopy data from the phase-separated $\mathrm{Si}(111) 5 \times 2$-Au surface (see Fig. 21), providing evidence for alternating metallic and semiconducting electronic structure. From [13].

the same origin. Likewise, a connection has recently been made between the Fermi surface and the chain length distribution for $\mathrm{Si}(553)-\mathrm{Au}$ [71].

A set of alternating metallic and insulating segments brings up the conceptual question, whether such a surface is a metal or an insulator. In fact, there has been a debate in the photoemission literature about this question. This could be settled by angle-resoled photoemission: If one finds two separate band structures for the metallic and semiconducting segments, the answer would be that the surface is both metallic and insulating. The scanning tunneling spectroscopy result suggests such a scenario. If the wave functions were delocalized over both segments, one would expect a single band structure with weakly metallic character.

There is an interesting application of the $\mathrm{Si}(111) 5 \times 2-\mathrm{Au}$ structure as atomic scale memory, where a bit is stored by the presence or absence of an extra $\mathrm{Si}$ atom [75]. The chains are used as self-assembled tracks that are precisely five atom rows wide. The storage density is comparable to that of DNA ( 1 bit per $5 \times 4=20$ atoms versus 32 atoms per bit for DNA). If one wants to apply Moore's law to estimate the arrival of such a technology, one can scale the density up to 250 Terabits/inch ${ }^{2}$ or the track width down to $1.6 \mathrm{~nm}$ and finds that this is expected to happen around 2040. Moore's law would have to remain valid twice as long as it has been in operation. Such a memory is highly impractical today, but allows testing the fundamental limits of data storage (see [75] for details). The density limit is actually due to the repulsive interaction between extra $\mathrm{Si}$ atoms discussed above, which makes it 
difficult putting two Si atoms into adjacent $5 \times 2$ cells and thereby doubling the density.

\subsection{The Puzzle of Two Half-Filled Bands: Spin-Splitting?}

The observation of two half-filled bands brings up an intriguing question: Why does the surface choose two half-filled bands (corresponding to two broken bonds) instead of one completely-filled band (corresponding to a covalent bond). An early explanation associated the splitting with spin-charge separation [45], one of the exotic phenomena predicted for 1D electrons. However, this option is ruled out by the fact that the splitting does not vanish at $E_{F}$ (see [77] and Fig. 23, right). Another argument against spin-charge separation is the band filling, which adds up to an even number of electrons per atom for $\mathrm{Si}(557)-\mathrm{Au}$, while spin-charge separation would predict an odd number.

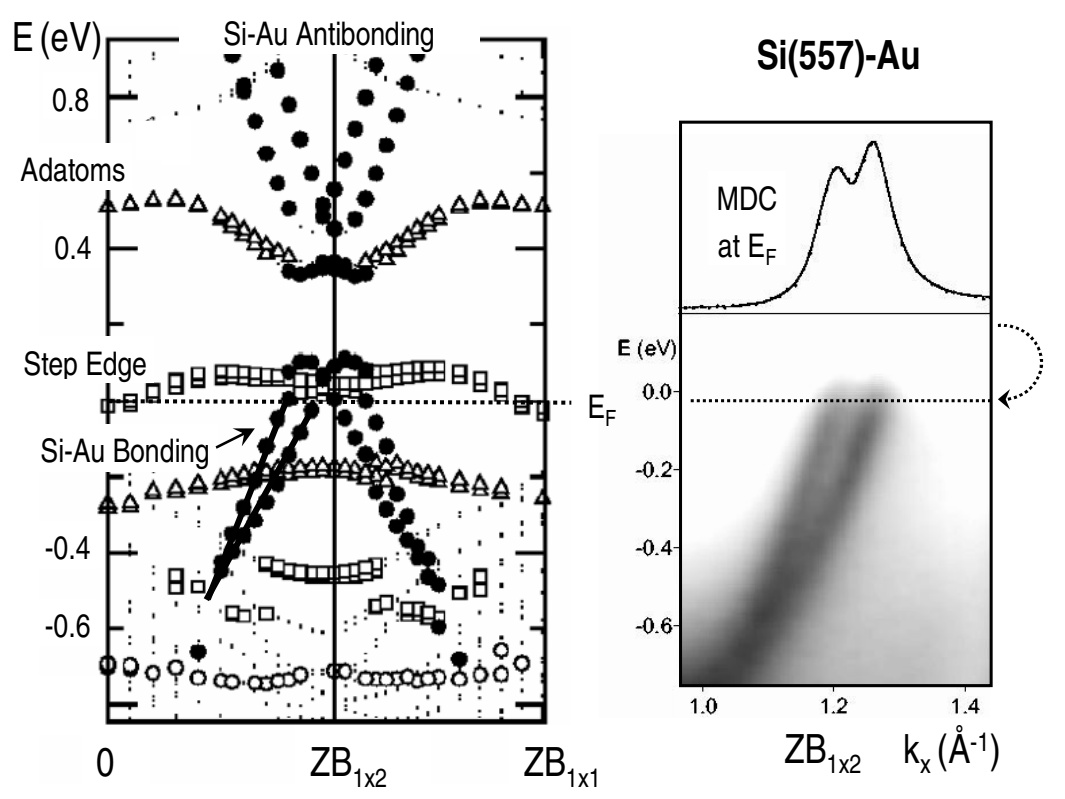

Fig. 23. Comparison between band calculations (left, from [78]) and angle-resolved photoemission (right, from [77]) for the $\mathrm{Si}(557)-\mathrm{Au}$ chain structure. In both cases, a nearly-degenerate doublet of bands crosses the Fermi level at $Z B_{1 x 2}$, the Brillouin zone boundary of a $1 \times 2$ cell (doubled along the chain direction by Si adatoms). The calculation shows back-folding at the $1 \times 2$ boundary, which is very weak in the experiment due to the weakness of the $1 \times 2$ lattice potential at the center of the terrace, where the Au chain resides.

This leaves several other potential explanations for the splitting, some of them rather exotic as well, such as a spin-splitting at a non-magnetic surface. First, consider the orbitals surrounding each Au atom. Fig. 20 gives 
a structural model for the Au chain, which applies to both $\mathrm{Si}(557)-\mathrm{Au}$ and $\mathrm{Si}(553)-\mathrm{Au}$. Although Au is coordinated with three $\mathrm{Si}$ atoms underneath, it is monovalent and forms only a single covalent bond. By symmetry, one can assume this to be the bond to the left in Fig. 20. The two remaining Si atoms on the right point towards the $\mathrm{Au}$ with broken bond orbitals. These have $p_{x}+p_{y}$, $p_{x}-p_{y}$ character and thus form a doubly-degenerate state. The degeneracy can be removed by the formation of bonding and antibonding combinations. In fact, a local density calculation [?] predicts such a band (full circles in Fig. 23 left). The bonding-antibonding splitting creates a gap of about $0.2 \mathrm{eV}$ at the zone boundary $Z B_{1 \times 2}$.

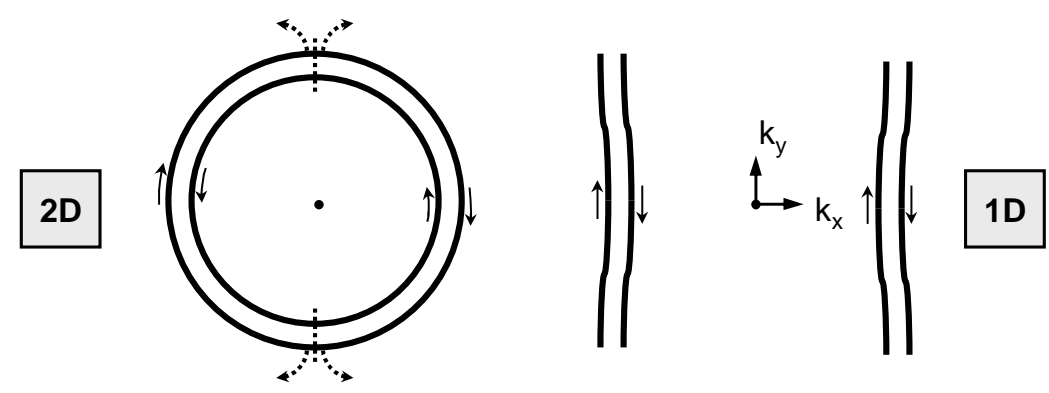

Fig. 24. Schematic of the spin splitting of the Fermi surface in $2 \mathrm{D}$ and $1 \mathrm{D}$, induced by spin-orbit interaction. The prototypical 2D case is $\mathrm{Au}(111)$ (left, see $[33,34]$ ), where the Rashba Hamiltonian shows how the lack of inversion symmetry about the surface lifts the spin-degeneracy. The $2 \mathrm{D}$ Fermi surface can be converted into $1 \mathrm{D}$ by cutting it in two places and deforming it into the 1D Fermi surface observed on $\mathrm{Si}(557)-\mathrm{Au}$ and $\mathrm{Si}(553)-\mathrm{Au}$ (see compare Fig. 19). This explains the spin splitting predicted for $\mathrm{Si}(557)$ [78] (see the left panel of Fig. 23).

The actual splitting of the band in Fig. 23, however, is a spin splitting created by the spin-orbit interaction. This model reproduces the two bands seen in photoemission remarkably well when taking into account that the back-folded part of the band between $Z B_{1 \times 2}$ and $Z B_{1 \times 1}$ is hardly visible in photoemission. Spin-orbit interaction with heavy elements, such as $\mathrm{Au}$, is able to produce spin polarization at the surface of a non-magnetic material. While the net spin polarization integrated over the Brillouin zone remains zero, individual parts of the Brillouin zone become 100\% spin-polarized. Figure 24 shows a a schematic for a well-studied two-dimensional case [33, 34] where a single surface state band on $\mathrm{Au}(111)$ splits into two spin-split bands at the Fermi surface. These exhibit circular spin structures rotating in opposite directions. These two-dimensional Fermi circles can be converted into onedimensional Fermi lines by cutting them and deforming them into lines. The resulting one-dimensional spin pattern for the spin-split band on $\mathrm{Si}(557)-\mathrm{Au}$ contains opposite spins running up and down the Fermi lines. The splitting is actually larger for $\mathrm{Si}(557)-\mathrm{Au}$ than for $\mathrm{Au}(111)$. A critical test of this spin- 
orbit model for the band splitting would be spin-polarized, angle-resolved photoemission or the detection of dichroism with circularly-polarized light. The recently-observed pattern of band crossings at the $Z B_{1 \times 2}$ is consistent with the spin-polarized model (crossings between equal spins are avoided, but not between opposite spins [79]).

The band model in Fig. 23 left is rather promising, but several loose ends remain. The band filling of the spin-split bands is odd and leads to an odd overall electron count per Au atom, a problem already encountered with the spincharge separation model. The electron count cannot be changed by adding or removing $\mathrm{Si}$ atoms from the surface since each $\mathrm{Si}$ has four valence electrons. Bringing in electrons from the bulk would require the Fermi level to lie at or below the valence band maximum, which is not observed experimentally $\left(E_{F} \approx \mathrm{VBM}+0.1 \mathrm{eV}\right.$ at these surfaces). A second unsolved question is the existence of a flat band that straddles $E_{F}=0$ in Fig. 23 and becomes occupied near the $\Gamma$-point. It has not been observed in photoemission, but it would explain spectroscopic STM imaging, where metallic states are found near the step edge [80, 81, 82].

The empty part of the calculated surface band structure is completely different from the occupied part. Instead of a steep, free-electron-like band with a small effective mass of about 0.5 , one finds a flat band near $E_{F}$. At slightly higher energy there is even a band gap due to the large bonding/antibonding splitting of the $\mathrm{Si}-\mathrm{Au}$ orbitals. These states are just being explored by twophoton photoemission (see Section 4).

\subsection{Peierls Gap and Charge Density Waves}

A characteristic feature of one-dimensional systems is the Peierls distortion. Using rather generic arguments of trading off strain energy cost and electronic energy gain, one can show that a one-dimensional system with a half-filled, metallic band generally doubles its period at low enough temperatures. The doubled lattice period induces a mini-gap at the Fermi level, which lowers the energy of the occupied states and leads to a metal-insulator transition. Although there may be exceptions from this generic case (for example in multiple chains or carbon nanotubes), the threat of a Peierls transition prevents searching for exotic 1D phenomena at very low temperatures. A better strategy consists of raising the transition temperature for the interesting phases, such as superconductivity and spin-charge separation, such that they can compete with the Peierls transition. This may be achieved by tailoring the interactions such that the electrons become increasingly correlated (for the case of spin-charge separation see the section by Claessen et al.).

Despite the fact that atom chains at semiconductor surfaces are firmly embedded into the substrate lattice, they seem not to be completely immune against the formation of Peierls-like gaps. Angle-resolved photoemission finds that the metallic bands gradually open up mini-gaps at $E_{F}$ in the temperature range between $300 \mathrm{~K}$ and $100 \mathrm{~K}$ (see Fig. 25 and $[83,73]$ ). Even the 

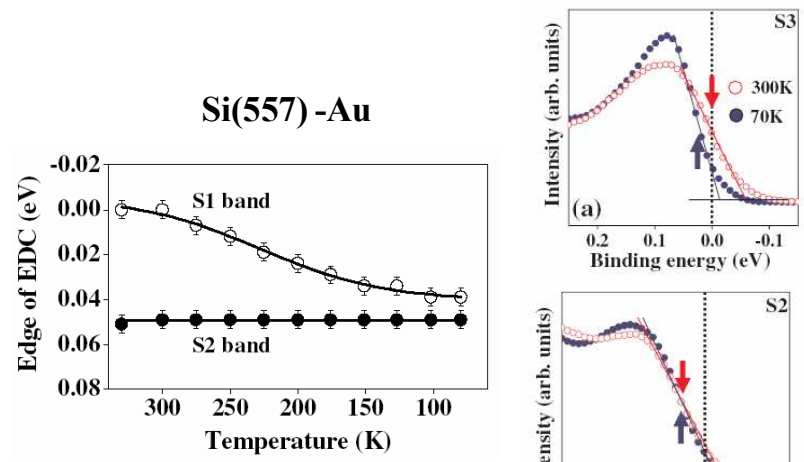

$\operatorname{Si}(553)-A u$
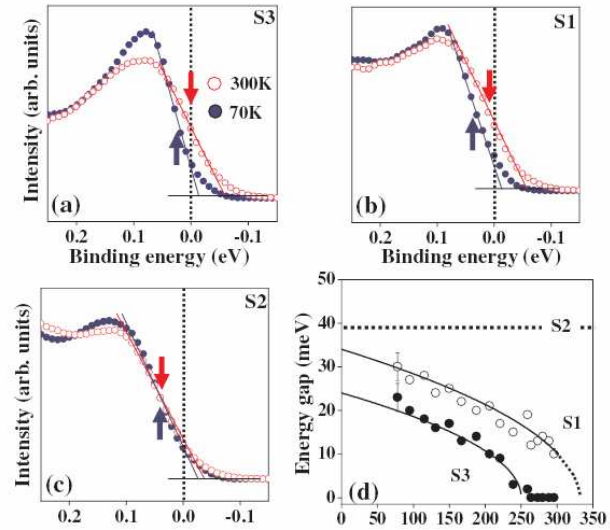

Fig. 25. Formation of a Peierls-like gap in the one-dimensional, metallic bands of $\mathrm{Si}(557)-\mathrm{Au}$ and $\mathrm{Si}(553)-\mathrm{Au}$. From [83, 73]

fractionally-filled bands show such a gap. The formation of mini-gaps is accompanied by period doubling (tripling for the fractionally-filled band). These effects have been studied extensively by comparing angle-resolved photoemission and scanning tunneling spectroscopy [43, 83, 44, 73, 72].
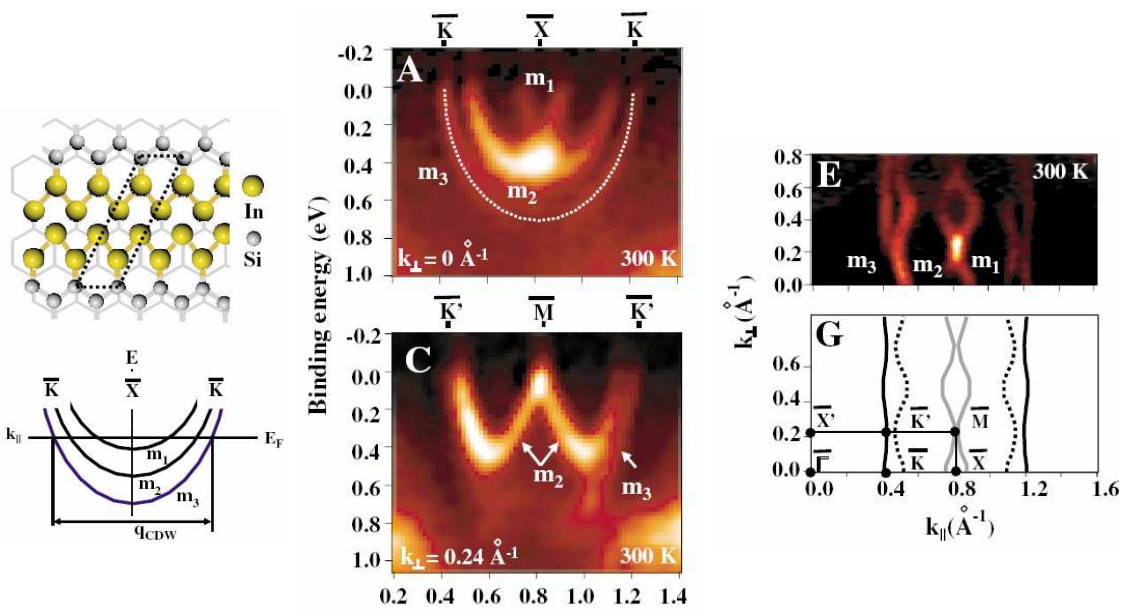

Fig. 26. Multiple metallic bands and interacting charge density waves in the fourchain structure $\mathrm{Si}(111) 5 \times 4$-In. From [44].

Observing period doubling by scanning tunneling microscopy does not necessarily imply a lattice distortion, as in the Peierls scenario. Charge density 
waves are able to modulate STM images without any atomic displacements. They are driven purely by the instability of parallel ("nested") Fermi surfaces towards the opening of a gap at $E_{F}$. One-dimensional Fermi surfaces are straight lines and, thus, always perfectly nested.

Multiple chain structures can give rise to multiple charge density waves with different periods. That opens up a rich area of interacting atomic chains and the charge density waves associated with them. An example is the fourchain structure of the $\mathrm{Si}(111) 5 \times 4$-In surface in Fig. 26 .

\section{Summary and Future Avenues}

There is one key message to be taken away from this overview: Atomic chains at surfaces provide a new playground for low-dimensional physics. These structures can be tailored systematically, which makes it possible to explore a large part of the parameter space of one-dimensional phase diagrams. The dimensionality, for example, is varied via the step spacing from 2D to $1 \mathrm{D}$. Metallic chains on semiconducting substrates can be completely de-coupled from the bulk electronically, while locking the chain atoms firmly in place by backbonds. Spin chains can be created by transition metal and rare earth atoms. Magnetic Co chains exhibit a dramatic increase of the orbital moment.

Atomic chains on noble metal surfaces tend to derive their electronic structure from free-electron-like 2D surface states, which makes them prototypes for demonstrating the concepts of low-dimensional electronic states. Various types of confinement lead to states confined to terraces, to step states, and to superlattice states which propagate across steps and terraces. It has become possible to determine these wave functions directly from a Fourier transformation of the momentum distribution observed by photoemission, using phase recovery methods from x-ray optics.

All kinds of unexpected, sometimes counter-intuitive phenomena appear in chains on semiconductor substrates. Chains of metal atoms do not attach themselves to step edges, as expected from step-flow growth and observed on metal surfaces. They stay right in the middle of the terrace. The step edge is taken over by the honeycomb chain at $\mathrm{Si}(111)$ surfaces, a graphitic form of silicon stabilized by $\pi$-bonding, which is highly unstable in Si compounds. In fact, this graphitic strip of silicon may be the driving force for stabilizing 1D over 2D structures. Fractional band filling is seen for $\mathrm{Si}(553)-\mathrm{Au}$ and explained by indirect doping of the chains at the center of the terrace by $\mathrm{Si}$ dopants at the step edge. Nanoscale phase separation takes place by bunching of dopants and leads to alternation sections of metallic and semiconducting chain segments, a one-dimensional prototype for the two-dimensional stripes in high temperature superconductors.

This field is still wide open. Many of the unusual phenomena are still unexplained, and most of the structures have yet to be determined. Examples are the two half-filled bands of Au-on-Si(111) structures, which have led to 
proposals of spin-charge separation and spin-polarized bands at a surface composed of non-magnetic materials. Even the origin of the metallicity of atom chains on semiconductors is still in question. Do the metallic electrons come from the metal chains at the center of the terrace or from the graphitic $\mathrm{Si}$ chain at the edge?

Angle-resolved photoemission needs to be pushed to its limits by these structures, as far as momentum resolution is concerned. The unit cells in real space are rather large, in order to de-couple the chains, and that leads to tiny Brillouin zones. In the future, the angle-resolved photoemission with high spatial resolution may become an important tool for zooming in on small, but highly-perfect regions of a surface. For example, optical spectra from self-assembled quantum dots on semiconductors become increasingly sharper when focusing a laser down to the micron regime [?]. The ultimate goal would be angle-resolved photoemission from single nano-objects, such as a quantum dot or a nanotube. The size distribution would be eliminated completely. The wave-function reconstruction techniques developed for confined terrace states on metals are well-matched to such systems.
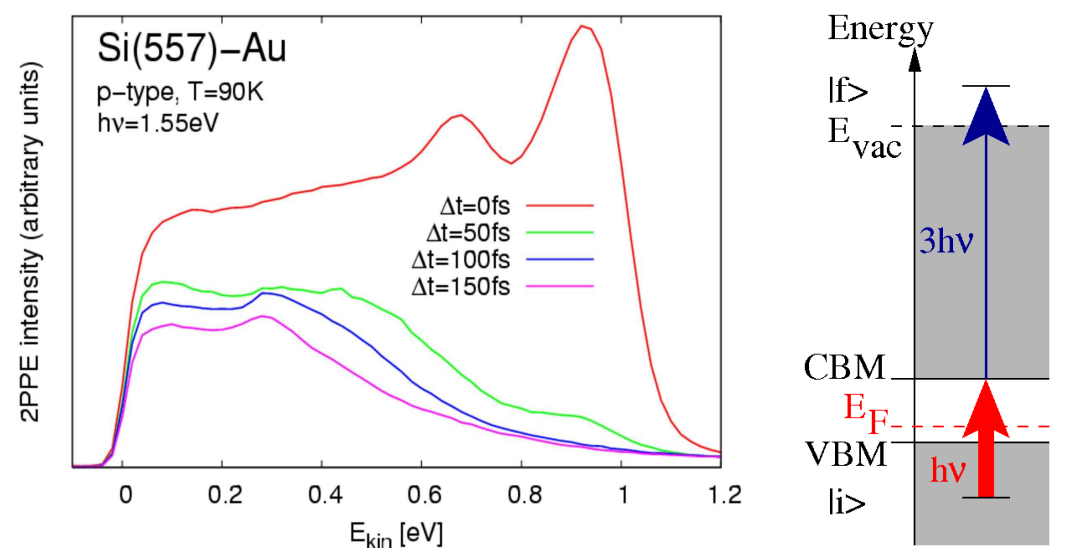

Fig. 27. Two-photon photoemission as future avenue for probing the unoccupied states of low-dimensional surface structures and determining the dynamics of hot electrons. The data are for $\mathrm{Si}(557)-\mathrm{Au}$, where the position of the lowest peak shifts down in 50-150 fs, as the hot electrons relax to the bottom of the conduction band. From [84].

Isolating the photemission signal of such nano-objects from the substrate background is going to be quite a challenge. The cross section can be optimized for specific wave functions by varying the photon energy and the polarization using synchrotron radiation. For example, the one-dimensional states on $\mathrm{Si}$ surfaces become most pronounced in the magic photon energy range $34-40 \mathrm{eV}$, while being rather weak at the HeI resonance line. Resonant photoemission 
enhances a specific element, while a Cooper minimum can be used to suppress the substrate.

Another challenge for angle-resolved photoemission is the connection to transport measurements, such as conductivity and superconductivity of surfaces. These measurements become very difficult to apply to atomic chains, let alone a single atom chain. Photoemission measures the complete set of quantum numbers for surface electrons, and the resulting set of energy bands and linewidths could conceivably be used to calculate transport from photoemission data. That will require a very high energy resolution, which has to be small compared to the thermal energy $k_{B} T$.

While photoemission does an excellent job with occupied electronic states, it does not provide information about unoccupied states between the Fermi level and the vacuum level - apart from a small energy slice above the Fermi edge that is populated by thermal electrons. Inverse photoemission is the natural counterpart to photoemission, but the extremely low cross section of this technique has not allowed it to reduce the energy resolution below thermal energies. There is another option for accessing unoccupied states with high energy resolution, as long as they are long-lived: That is two-photon photoemission, where the first photon pumps electrons into an unoccupied state and the second photon ionizes them. This technique is just beginning to be applied to atomic chain structures, as shown in Fig. 27 [84]. The schematic diagram on the right illustrates the process. By combining high intensity infrared and low-intensity UV photons ( $h \nu$ and $3 h \nu)$ one avoids swamping the analyzer with low energy electrons from the thermal tail above $E_{F}$ excited by single UV photons. The sequence of events can be determined by delaying the UV with respect to the IR pulse or vice versa. This type of spectroscopy does not only provide energy bands, it also follows the relaxation of the excited electrons in real time. In Fig. 27, the electrons from the low-energy peak gradually lose their kinetic energy over 50-150 femtoseconds. These initial results suggest that there will be a lot more information available in the near future to solve the many riddles of atomic chains at surfaces.

\section{References}

1. E. H.Libe, D. C. Mattis (eds.) in: Mathematical Physics in One Dimension: Exactly Soluble Models of Interacting Particles (Academic, New York, 1966)

2. T. Giamarchi in: Quantum Physics in One Dimension (Oxford University Press, New Nork, 2004).

3. J. Solyom: Adv. Phys. 28, 201 (1979).

4. K. Schönhammer in: Strong Interactions in Low Dimensions, ed. by D. Baeriswyl and L. Degiorgi (Klumer Academic Publishers, 2003) Ch.1 Section 5.2 .

5. J. Voit: Rep. Prog. Phys. 58, 977 (1995).

6. G. Gruner: Density Waves in Solids (Perseus Publishing, Cambridge, Massachusetts 1994). 
7. P. M. Chaikin, E. I. Chashechkina, I. J. Lee, and M. J. Naughton: J. Phys.Condes. Matter 10, 11301 (1998).

8. H. Ohnishi, Y. Kondo and K. Takayanagi: Nature 395, 780 (1998).

9. V. Rodrigues, T. Fuhrer, and D. Ugarte: Phys. Rev. Lett. 85, 4124 (2000).

10. J. N. Crain and F. J. Himpsel, Appl. Phys. A 82, 431 (2006).

11. J. N. Crain, A. Kirakosian, K. N. Altmann, C. Bromberger, S. C. Erwin, J. L. McChesney, J. L. Lin, and F. J. Himpsel: Phys. Rev. Lett. 90, 176805 (2003).

12. J. N. Crain, J. L. McChesney, Fan Zheng, M. C. Gallagher, P. C. Snijders, M. Bissen, C. Gundelach, S. C. Erwin, and F. J. Himpsel: Phys. Rev. B 69, 125401 (2004).

13. H. S. Yoon, S. J. Park, J. E. Lee, C. N. Whang, and I.-W. Lyo: Phys. Rev. Lett. 92, 096801 (2004).

14. A. Vindigni, A. Rettori, M. G. Pini, C. Carbone, and P. Gambardella: Appl. Phys. A 82, 385 (2006).

15. A. Kirakosian, J. L. McChesney, R. Bennewitz, J. N. Crain, J.-L. Lin, and F. J. Himpsel: Surf. Sci. 498, L109 (2002).

16. J. N. Crain, K. N. Altmann, C. Bromberger, and F. J. Himpsel: Phys. Rev. B 66, 205302 (2002).

17. J.-L. Lin, Y. Petrovykh, A. Kirakosian, H. Rauscher, F. J. Himpsel, and P. A. Dowben: Appl. Phys. Lett. 78, 829 (2001).

18. N. Papageorgiou, Y. Ferro, J. M. Layet, L. Giovanelli, A. J. Mayne, G. Dujardin, H. Oughaddou, and G. Le Lay: Appl. Phys. Lett. 82, 2518 (2003).

19. E. D. Williams: Surf. Sci. 299/300, 502 (1994).

20. A. R. Bachmann, A. Mugarza, J. E. Ortega, and S. Speller: Phys. Rev. B 64, 153409 (2001).

21. J. Lobo, E. G. Michel, A. Bachmann, S. Speller, J. Kuntze, and J. E. Ortega: Phys. Rev. Lett. 93, 137602 (2004).

22. J. E. Ortega, M. Ruiz-Osés, and J. Kuntze: Phys. Rev. B 72, 195416 (2005).

23. J. E. Ortega, M. Ruiz-Osés, J. Cordón, A. Mugarza, J. Kuntze, and F. Schiller: New Journal of Phys. 7, 101 (2005).

24. A. Mugarza and J. E. Ortega: J. Phys. Cond. Mat. 15, S3281 (2003).

25. A. Mugarza, F. Schiller, J. Kuntze, J. Cordón, M. Ruiz-Osés, and J. E. Ortega: J. of Phys. C 18, S27 (2006).

26. L. Bürgi, O. Jeandupeux, A. Hirstein, H. Brune, and K. Kern: Phys. Rev. Lett. 81, 5370 (1998).

27. A. Mugarza, J. E. Ortega , F. J. Himpsel, and F. J. García de Abajo: Phys. Rev. B 67, 081404 (2003).

28. J. Miao, P. Charalambous, J. Kirz, and D. Sayre: Nature (London) 400, 342 (1999).

29. M. Giesen, G. Schulze Icking-Konert, and H. Ibach: Phys. Rev. Lett. 82, 3101 (1999).

30. K. Morgenstern, K.-F. Braun, and K.-H. Rieder: Phys. Rev. Lett. 89, 226801 (2002).

31. F. Baumberger, M. Hengsberger, M. Muntwiler, M. Shi, J. Krempasky, L. Patthey, J. Osterwalder, and T. Greber: Phys. Rev. Lett. 92, 16803 (2004).

32. A. Mugarza, A. Mascaraque, V. Repain, S. Rousset, K. N. Altmann, F. J. Himpsel, Yu. M. Koroteev, E. V. Chulkov, F. J. García de Abajo, and J. E. Ortega: Phys. Rev. B 66, 245419 (2002).

33. S. LaShell, B. A. McDougall, and E. Jensen, Phys. Rev. Lett. 77, 3419 (1996). 
34. F. Reinert, G. Nicolay, S. Schmidt, D. Ehm, and S. Hüfner: Phys. Rev. B 63, 115415 (2001).

35. F. Schiller, J. Cordón, M. Ruiz-Osés, and J. E. Ortega: Phys. Rev. Lett. 95, 066805 (2005).

36. M. Roth, M. Pickel, W. Jinxiong, M. Weinelt, and T. Fauster: Phys. Rev. Lett. 88, 096802 (2002).

37. S. Smadici and R. M. Osgood: Phys. Rev. B 71, 165424 (2005).

38. F. Schiller, J. Cordón, D. Vyalikh, A. Rubio, and J. E. Ortega: Phys. Rev. Lett. 94, 016103 (2005).

39. R. Eder and H. Winter: Phys. Rev. B 70, 085413 (2004).

40. X. Y. Wang, X. J. Shen, and R. M. Osgood, Jr.: Phys. Rev. B 56, 7665 (1997).

41. S. Hasegawa, X. Tong, S. Takeda, N. Sato and T. Nagao, Prog. Surf. Sci. 60, 89 (1999).

42. J. N. Crain, M. C. Gallagher, J. L. McChesney, M. Bissen, and F. J. Himpsel: Phys. Rev. B 72, 045312 (2005).

43. H. W. Yeom, S. Takeda, E. Rotenberg, I. Matsuda, K. Horikoshi, J. Schaefer, C. M. Lee, S. D. Kevan, T. Ohta, T. Nagao, and S. Hasegawa: Phys. Rev. Lett. 82, 4898-4901 (1999).

44. J. R. Ahn, J. H. Byun, H. Koh, E. Rotenberg, S. D. Kevan, and H. W. Yeom: Phys. Rev. Lett. 93, 106401 (2004).

45. P. Segovia, D. Purdie, M. Hengsberger, and Y. Baer: Nature 402, 504 (1999).

46. A. Kirakosian, R. Bennewitz, J. N. Crain, Th. Fauster, J.-L. Lin, D. Y. Petrovykh, and F.J. Himpsel: Appl. Phys. Lett. 79, 1608 (2001).

47. J. Kuntze, A. Mugarza, and J. E. Ortega: Appl. Phys. Lett. 81, 2463 (2002).

48. S. C. Erwin and H. H. Weitering: Phys. Rev. Lett. 81, 2296 (1998).

49. D. Y. Petrovykh, K. N. Altmann, J.-L. Lin, F. J. Himpsel, and F. M. Leibsle: Surf. Sci. 512, 269 (2002).

50. R. Losio, K. N. Altmann, and F. J. Himpsel: Phys. Rev. Lett. 85, 808 (2000).

51. S. C. Erwin: Phys. Rev. Lett. 91, 206101 (2003)

52. J. L. McChesney, J. N. Crain, V. Pérez-Dieste, F. Zheng, M. C. Gallagher, M. Bissen, C. Gundelach, and F. J. Himpsel: Phys. Rev. B 70, 195430 (2004).

53. J. Viernow, J. L. Lin, D. Y. Petrovykh, F. M. Leibsle, F. K. Men, and F. J. Himpsel: Appl. Phys. Lett. 72, 948 (1998).

54. J.-L. Lin, D. Y. Petrovykh, J. Viernow, F. K. Men, D. J. Seo, and F. J. Himpsel: J. Appl. Phys. 84, 255 (1998).

55. D. Sánchez-Portal and R. M. Martin: Surf. Sci. 532, 655 (2003).

56. S. C. Erwin: Phys. Rev. Lett. 91, 206101 (2003).

57. S. C. Erwin: unpublished.

58. S.C. Erwin and H.H. Weitering: Phys. Rev. Lett. 81, 2296 (1998).

59. I. K. Robinson, P. A. Bennett, and F. J. Himpsel: Phys. Rev. Lett. 88, 096104 (2002).

60. D. Sánchez-Portal, J. D. Gale, A. Garcia, and R. M. Martin: Phys. Rev. B 65, 081401 (2002).

61. R. I. G. Uhrberg, H. M. Zhang, T. Balasubramanian, E. Landemark, H. W. Yeom: Phys. Rev. B 65, 081305(R) (2002).

62. Y. G. Ding, C. T. Chan, and K. M. Ho: Phys. Rev. Lett. 67, 1454 (1991).

63. H. Aizawa and M. Tsukada: Phys. Rev. B 59, 10923 (1999).

64. R. Losio, K. N. Altmann, and F. J. Himpsel: Phys. Rev. B 61, 10845 (2000).

65. J. Ortega, F. Flores, and A. L. Yeyati: Phys. Rev. B 58, 4584 (1998). 
66. F. Flores, A. L. Yeyati, and J. Ortega: Surf. Rev. Lett. 4, 281 (1997).

67. J.E. Demuth, B.N.J. Persson, and A. Schell-Sorokin: Phys. Rev. Lett. 51, 2214 (1983).

68. R. Schillinger, C. Bromberger, H. J. Jnsch, H. Kleine, O. Khlert, C. Weindel, and D. Fick: Phys. Rev. B 72, 115314 (2005).

69. I. Barke, Fan Zheng, A. R. Konicek, R. C. Hatch, and F. J. Himpsel, Phys. Rev. Lett., in press (2006).

70. J. N. Crain and D. T. Pierce: Science 307, 703 (2006).

71. J. N. Crain, M. D. Stiles, J. A. Stroscio, and D. T. Pierce: Phys. Rev. Lett. 96, 156801. (2006).

72. P. C. Snijders, S. Rogge, and H. H. Weitering: Phys. Rev. Lett. 96, 076801 (2006).

73. J. R. Ahn, P. G. Kang, K. D. Ryang, and H. W. Yeom: Phys. Rev. Lett. 95, $196402(2005)$

74. F. J. Himpsel, J. L. McChesney, J. N. Crain, A. Kirakosian, V. Pérez-Dieste, Nicholas L. Abbott, Yan-Yeung Luk, Paul F. Nealey, Dmitri Y. Petrovykh: J. Phys. Chem. B 108, 14484 (2004).

75. R. Bennewitz, J. N. Crain, A. Kirakosian, J.-L. Lin, J. L. McChesney, D. Y. Petrovykh, and F. J. Himpsel: Nanotechnology 13, 499 (2002).

76. A. Kirakosian, R. Bennewitz, F. J. Himpsel, and L. W. Bruch: Phys. Rev. B 67, 205412 (2003).

77. R. Losio, K. N. Altmann, A. Kirakosian, J.-L. Lin, D. Y. Petrovykh, and F. J. Himpsel: Phys. Rev. Lett. 86, 4632 (2001).

78. D. Sánchez-Portal, S. Riikonen, and R. M. Martin: Phys. Rev. Lett. 93, 146803 (2004).

79. I. Barke et al.: unpublished.

80. H. W. Yeom, J. R. Ahn, H. S. Yoon, I.-W. Lyo, H. Jeong, and S. Jeong: Phys. Rev. B 72, 035323 (2005).

81. M. Schöck, C. Sürgers, and H. v. Löhneisen: Europhys. Lett. 74, 473 (2006).

82. M. Krawiec, T. Kwapinski, and M. Jalochowski: Phys. Rev. B 73, 075415 (2006).

83. J. R. Ahn, H. W. Yeom, H. S. Yoon, and I.-W. Lyo: Phys. Rev. Lett. 91, 196403 (2003).

84. T. K Rügheimer, et al., to be published. 